\title{
Cobordism of Morse functions on surfaces, the universal complex of singular fibers and their application to map germs
}

\author{
OSAMU SAEKI
}

\begin{abstract}
We give a new and simple proof for the computation of the oriented and the unoriented fold cobordism groups of Morse functions on surfaces. We also compute similar cobordism groups of Morse functions based on simple stable maps of 3-manifolds into the plane. Furthermore, we show that certain cohomology classes associated with the universal complexes of singular fibers give complete invariants for all these cobordism groups. We also discuss invariants derived from hypercohomologies of the universal homology complexes of singular fibers. Finally, as an application of the theory of universal complexes of singular fibers, we show that for generic smooth map germs $g:\left(\mathbb{R}^{3}, 0\right) \rightarrow\left(\mathbb{R}^{2}, 0\right)$ with $\mathbb{R}^{2}$ being oriented, the algebraic number of cusps appearing in a stable perturbation of $g$ is a local topological invariant of $g$.
\end{abstract}

57R45; 57R75, 58K60, 58K65

\section{Introduction}

In [19], Rimányi and Szúcs introduced the notion of a cobordism for singular maps. In fact, in the classical work of Thom [27], one can find the notion of a cobordism for embeddings, and they naturally generalized this concept to differentiable maps with prescribed local and global singularities. In particular, when the dimension of the target manifold is greater than or equal to that of the source manifold, they described the cobordism groups in terms of the homotopy groups of a certain universal space by means of a Pontrjagin-Thom type construction.

However, when the dimension of the target is strictly smaller than that of the source, their method cannot be directly applied. In [22], the author defined the cobordism group for maps with only definite fold singularities, and used a geometric argument in [24] to show that the cobordism group of such functions is isomorphic to the $h-$ cobordism group of homotopy spheres (Kervaire-Milnor [11]). Recently this result was generalized for maps by Sadykov [20] with the aid of a Pontrjagin-Thom type construction. This was possible, since the class of singularities is quite restricted and the structure of regular fibers of such maps is well-understood. 
On the other hand, Ikegami and the author [8] defined and studied the oriented (fold) cobordism group of Morse functions on surfaces. Since a (fold) cobordism between Morse functions on closed surfaces does not allow cusp singularities, this cobordism group is rather bigger than the usual cobordism group of 2-dimensional manifolds. In fact, Ikegami and the author employed a geometric argument using functions on finite graphs to show that the group is in fact an infinite cyclic group. Recently, the structure of the unoriented cobordism group of Morse functions on surfaces was determined by Kalmár [9] by using a similar method. In [7] Ikegami determined the structures of the oriented and the unoriented cobordism groups of Morse functions on manifolds of arbitrary dimensions by using an argument employing the cusp elimination technique based on Levine [13].

On the other hand, in an attempt to construct a rich family of cobordism invariants for maps with prescribed local and global singularities in the case where the dimension of the target is strictly smaller than that of the source, the author considered singular fibers of such maps and developed the theory of universal complexes of singular fibers [25]. The terminology "singular fiber" here refers to a certain right-left equivalence class of a map germ along the inverse image of a point in the target. The equivalence classes of such singular fibers together with their adjacency relations lead to a cochain complex, and in [25] it was shown that its cohomology classes give rise to cobordism invariants for singular maps. In fact, the isomorphism between the oriented cobordism group of Morse functions on surfaces and the infinite cyclic group was reconstructed by using an invariant derived from the universal complex of singular fibers [25, Section 14.2].

This paper has three purposes. The first one (see Sections 2 and 3) is to give a new and simple proof for the calculation of the oriented and the unoriented fold cobordism groups of Morse functions on surfaces (Theorem 2.4). In Ikegami-Saeki [8] and Kalmár [9], the calculation was done by simplifying a given function on a graph by employing certain moves. In this paper, we also use the same moves, but the simplification is drastically simple.

Furthermore, we will introduce the notion of the oriented and the unoriented simple fold cobordism groups of Morse functions on surfaces by restricting the fold cobordisms to simple ones (for simple maps, the reader is referred to [21; 23] or Definition 2.5 of the present paper). We will show that the simple cobordism groups are in fact isomorphic to the corresponding cobordism groups (Theorem 2.7).

The second purpose of this paper is to construct cobordism invariants for the four cobordism groups of Morse functions on surfaces considered above by using cohomology classes of the universal complexes of singular fibers as developed in [25] (see Sections 4 and 5). By combining the universal complex of co-orientable singular fibers (with 
coefficients in $\mathbb{Z}$ ) and that of usual (not necessarily co-orientable) singular fibers (with coefficients in $\mathbb{Z}_{2}$ ), we will obtain complete cobordism invariants for all the four cases above.

In [10], Kazarian constructed the universal homology complex of singularities by combining the universal complex of co-orientable singularities and that of usual (not necessarily co-orientable) singularities defined by Vassiliev [28], and studied their hypercohomologies. In Section 5, we will consider the analogy of Kazarian's construction in our situation of singular fibers. We will see that the hypercohomology classes give rise to cobordism invariants, but for cobordism groups of Morse functions on surfaces, we obtain the same invariants as those obtained by using the usual universal complex of singular fibers. It would be interesting to study the hypercohomologies of higher dimensional analogues to see if there is a "hidden singular fiber" in a sense similar to Kazarian's [10].

The third purpose of this paper is to give an application of the theory of universal complexes of singular fibers developed in Sections 4 and 5 to the theory of stable perturbations of map germs. More precisely, we will consider a smooth map germ $g:\left(\mathbb{R}^{3}, 0\right) \rightarrow\left(\mathbb{R}^{2}, 0\right)$ which is generic in the sense of Fukuda and Nishimura $[3 ; 15]$. Then a stable perturbation $\tilde{g}$ of a representative of $g$ has isolated cusps, and for each cusp singular point we can define a sign +1 or -1 by using the indices of the nearby fold points together with the orientation of the target $\mathbb{R}^{2}$. Then by using the theory of singular fibers (of generic maps of 3-dimensional manifolds with boundary into the 2-dimensional disk), we will show that the algebraic number of cusps of $\widetilde{g}$ is a local topological invariant of $g$ with $\mathbb{R}^{2}$ being oriented (Theorem 6.3). We also describe this integer by a certain cobordism invariant of a $C^{\infty}$ stable map of a compact surface with boundary into $S^{1}$ associated with $g$. This invariant is strongly related to the isomorphism between the oriented cobordism group of Morse functions on surfaces and the infinite cyclic group constructed in Sections 3 and 4. It would be interesting to compare our result with those obtained by Fukuda and Ishikawa [4], Fukui, Nuño Ballesteros and Saia [5], Nuño Ballesteros and Saia [16], and Ohsumi [18], about the number of certain singularities appearing in a stable perturbation (or a generic deformation) of a given map germ.

Throughout the paper, manifolds and maps are differentiable of class $C^{\infty}$ unless otherwise indicated. For a topological space $X, \operatorname{id}_{X}$ denotes the identity map of $X$.

The author would like to thank Boldizsár Kalmár and Toshizumi Fukui for stimulating discussions. The author has been supported in part by Grant-in-Aid for Scientific Research (No. 16340018), Japan Society for the Promotion of Science. 


\section{Preliminaries}

In this section, we recall some basic notions about smooth functions and maps, and state two theorems about cobordism groups of Morse functions on surfaces.

A smooth real-valued function on a smooth manifold is called a Morse function if its critical points are all non-degenerate. We do not assume that the values at the critical points are all distinct: distinct critical points may have the same value. If the critical values are all distinct, then such a Morse function is said to be $\left(C^{\infty}\right)$ stable. For details, see Golubitsky and Guillemin [6, Chapter III, Section 2].

For a positive integer $n$, we denote by $M^{S O}(n)$ (or $M(n)$ ) the set of all Morse functions on closed oriented (resp. possibly nonorientable) $n$-dimensional manifolds. We adopt the convention that the function on the empty set $\varnothing$ is an element of $M^{S O}(n)$ and of $M(n)$ for all $n$.

Before defining the cobordism groups of Morse functions, let us recall the notion of fold singularities. Let $f: M \rightarrow N$ be a smooth map between smooth manifolds with $n=\operatorname{dim} M \geq \operatorname{dim} N=p$. A singular point of $f$ is a point $q \in M$ such that the rank of the differential $d f_{q}: T_{q} M \rightarrow T_{f(q)} N$ is strictly smaller than $p$. We denote by $S(f)$ the set of all singular points of $f$ and call it the singular set of $f$. A singular point $q \in S(f)$ is a fold singular point (or a fold point) if there exist local coordinates $\left(x_{1}, x_{2}, \ldots, x_{n}\right)$ and $\left(y_{1}, y_{2}, \ldots, y_{p}\right)$ around $q$ and $f(q)$ respectively such that $f$ has the form

$$
y_{i} \circ f=\left\{\begin{array}{l}
x_{i}, \quad 1 \leq i \leq p-1, \\
\pm x_{p}^{2} \pm x_{p+1}^{2} \pm \cdots \pm x_{n}^{2}, \quad i=p .
\end{array}\right.
$$

If the signs appearing in $y_{p} \circ f$ all coincide, then we say that $q$ is a definite fold singular point (or a definite fold point), otherwise an indefinite fold singular point (or an indefinite fold point).

If a smooth map $f$ has only fold points as its singularities, then we say that $f$ is a fold map.

Definition 2.1 Two Morse functions $f_{0}: M_{0} \rightarrow \mathbb{R}$ and $f_{1}: M_{1} \rightarrow \mathbb{R}$ in $M^{S O}(n)$ are said to be oriented cobordant (or oriented fold cobordant) if there exist a compact oriented $(n+1)$-dimensional manifold $X$ and a fold map $F: X \rightarrow \mathbb{R} \times[0,1]$ such that

(1) the oriented boundary $\partial X$ of $X$ is the disjoint union $M_{0} \amalg\left(-M_{1}\right)$, where $-M_{1}$ denotes the manifold $M_{1}$ with the orientation reversed, and 
(2) we have

$$
\begin{aligned}
\left.F\right|_{M_{0} \times[0, \varepsilon)} & =f_{0} \times \operatorname{id}_{[0, \varepsilon)}: M_{0} \times[0, \varepsilon) \rightarrow \mathbb{R} \times[0, \varepsilon), \quad \text { and } \\
\left.F\right|_{M_{1} \times(1-\varepsilon, 1]} & =f_{1} \times \operatorname{id}_{(1-\varepsilon, 1]}: M_{1} \times(1-\varepsilon, 1] \rightarrow \mathbb{R} \times(1-\varepsilon, 1]
\end{aligned}
$$

for some sufficiently small $\varepsilon>0$, where we identify the open collar neighborhoods of $M_{0}$ and $M_{1}$ in $X$ with $M_{0} \times[0, \varepsilon)$ and $M_{1} \times(1-\varepsilon, 1]$ respectively.

In this case, we call $F$ an oriented cobordism between $f_{0}$ and $f_{1}$.

If a Morse function in $M^{S O}(n)$ is oriented cobordant to the function on the empty set, then we say that it is oriented null-cobordant.

It is easy to show that the above relation defines an equivalence relation on the set $M^{S O}(n)$ for each $n$. Furthermore, we see easily that the set of all oriented cobordism classes forms an additive group under disjoint union: the neutral element is the class corresponding to oriented null-cobordant Morse functions, and the inverse of a class represented by a Morse function $f: M \rightarrow \mathbb{R}$ is given by the class of $-f:-M \rightarrow \mathbb{R}$. We denote by $\mathcal{M}^{S O}(n)$ the group of all oriented (fold) cobordism classes of elements of $M^{S O}(n)$ and call it the oriented (fold) cobordism group of Morse functions on manifolds of dimension $n$, or the $n$-dimensional oriented cobordism group of Morse functions.

We can also define the unoriented versions of all the objects defined above by forgetting the orientations and by using $M(n)$ instead of $M^{S O}(n)$. For the terminologies, we omit the term "oriented" (or use "unoriented" instead) for the corresponding unoriented versions. The unoriented cobordism group of Morse functions on manifolds of dimension $n$ is denoted by $\mathcal{M}(n)$ by omitting the superscript $S O$.

Remark 2.2 The oriented cobordism group $\mathcal{M}^{S O}(n)$ is denoted by $\mathcal{M}(n)$ in IkegamiSaeki [8] and by $\mathcal{M}_{n}$ in Ikegami [7]. Furthermore, the unoriented cobordism group $\mathcal{M}(n)$ is denoted by $\mathcal{N}_{n}$ in [7] and by $\operatorname{Cob}_{f}(n, 1-n)$ in Kalmár [9].

Remark 2.3 Let $M$ be a closed (oriented) $n$-dimensional manifold. It is easy to see that if two Morse functions $f$ and $g$ on $M$ are connected by a one-parameter family of Morse functions, then they are (oriented) cobordant. In particular, every Morse function is (oriented) cobordant to a stable Morse function.

The following isomorphisms have been proved in $[7 ; 8 ; 9]$.

Theorem 2.4 (1) The 2-dimensional oriented cobordism group of Morse functions $\mathcal{M}^{S O}(2)$ is isomorphic to $\mathbb{Z}$, the infinite cyclic group. 
(2) The 2-dimensional unoriented cobordism group of Morse functions $\mathcal{M}(2)$ is isomorphic to $\mathbb{Z} \oplus \mathbb{Z}_{2}$.

In Section 3, we will give a new and simple proof for the above isomorphisms. We will also describe explicit isomorphisms.

In order to define new cobordism groups of Morse functions, we need the following.

Definition 2.5 Let $f: M \rightarrow N$ be a smooth map between smooth manifolds. We say that $f$ is simple if for every $y \in N$, each connected component of $f^{-1}(y)$ contains at most one singular point.

Note that a stable Morse function is always simple.

If $\operatorname{dim} N=2$ and $f: M \rightarrow N$ is a fold map which is $C^{\infty}$ stable (for details, see Golubitsky-Guillemin, Levine, Saeki $[6 ; 14 ; 25])$, then $S(f)$ is a regular 1dimensional submanifold of $M$ and the map $\left.f\right|_{S(f)}$ is an immersion with normal crossings. Therefore, for every $y \in N, f^{-1}(y)$ contains at most two singular points. Fold maps of closed 3-manifolds into the plane which are $C^{\infty}$ stable and simple have been studied by the author $[21 ; 23]$.

Definition 2.6 For a positive integer $n$, we denote by $S M^{S O}(n)$ (or $S M(n)$ ) the set of all stable Morse functions on closed oriented (resp. possibly nonorientable) $n$-dimensional manifolds.

Two stable Morse functions $f_{0}: M_{0} \rightarrow \mathbb{R}$ and $f_{1}: M_{1} \rightarrow \mathbb{R}$ in $S M^{S O}(n)$ are said to be simple oriented (fold) cobordant if there exist an oriented cobordism $F$ between $f_{0}$ and $f_{1}$ as in Definition 2.1 such that $F$ is simple and $\left.F\right|_{S(F)}$ is an immersion with normal crossings. In this case, we call $F$ a simple oriented cobordism between $f_{0}$ and $f_{1}$.

It is easy to show that the above relation defines an equivalence relation on the set $S M^{S O}(n)$ for each $n$. Furthermore, we see easily that the set of all simple oriented cobordism classes forms an additive group under disjoint union. We denote by $\mathcal{S M}^{S O}(n)$ the group of all simple oriented (fold) cobordism classes of elements of $S M^{S O}(n)$ and call it the simple oriented (fold) cobordism group of Morse functions on manifolds of dimension $n$, or the $n$-dimensional simple oriented cobordism group of Morse functions.

We can also define the unoriented versions of all the objects defined above. The simple unoriented cobordism group of Morse functions on manifolds of dimension $n$ is denoted by $\mathcal{S M}(n)$. 
We will prove the following in Section 3.

Theorem 2.7 (1) The 2-dimensional simple oriented cobordism group of Morse functions $\mathcal{S M}^{S O}(2)$ is isomorphic to $\mathbb{Z}$, the infinite cyclic group.

(2) The 2-dimensional simple unoriented cobordism group of Morse functions $\mathcal{S M}(2)$ is isomorphic to $\mathbb{Z} \oplus \mathbb{Z}_{2}$.

In fact, we will see that the natural homomorphisms

$$
\mathcal{S M}^{S O}(2) \rightarrow \mathcal{M}^{S O}(2) \text { and } \mathcal{S M}(2) \rightarrow \mathcal{M}(2)
$$

are isomorphisms.

\section{Proofs of the theorems}

In this section, we will prove Theorems 2.4 and 2.7.

Let us first recall the following notion of a Stein factorization (for more details, see $[12 ; 14 ; 25]$, for example).

Definition 3.1 Suppose that a smooth map $f: M \rightarrow N$ with $n=\operatorname{dim} M \geq \operatorname{dim} N=p$ is given. Two points in $M$ are equivalent with respect to $f$ if they lie on the same component of an $f$-fiber. Let $W_{f}$ denote the quotient space of $M$ with respect to this equivalence relation and $q_{f}: M \rightarrow W_{f}$ the quotient map. It is easy to see that then there exists a unique continuous map $\bar{f}: W_{f} \rightarrow N$ such that $f=\bar{f} \circ q_{f}$. The space $W_{f}$ or the commutative diagram

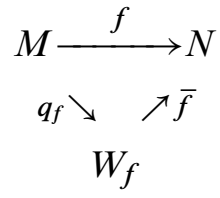

is called the Stein factorization of $f$.

If $f: M \rightarrow \mathbb{R}$ is a Morse function on a closed manifold $M$, then $W_{f}$ has the natural structure of a 1-dimensional CW complex. In this case, we call $W_{f}$ the Reeb graph of $f$ (for example, see [2]). Furthermore, we call the continuous map $\bar{f}: W_{f} \rightarrow \mathbb{R}$ a Reeb function.

Let $f: M \rightarrow \mathbb{R}$ be a stable Morse function on a closed (possibly nonorientable) surface $M$. Then by [8, Lemma 3.2] and [9, Section 3], its Reeb graph $W_{f}$ is a finite graph whose vertices are the $q_{f}$-images of the critical points of $f$ such that 
(1) the vertices corresponding to critical points of index 0 or 2 have degree 1 , and those of index 1 have degree 2 or 3 ,

and the Reeb function $\bar{f}: W_{f} \rightarrow \mathbb{R}$ satisfies the following:

(2) around each vertex of $W_{f}, \bar{f}$ is equivalent to one of the functions as depicted in Figure 1, and

(3) $\bar{f}$ is an embedding on each edge.

Furthermore, a degree 2 vertex occurs only if $M$ is nonorientable.
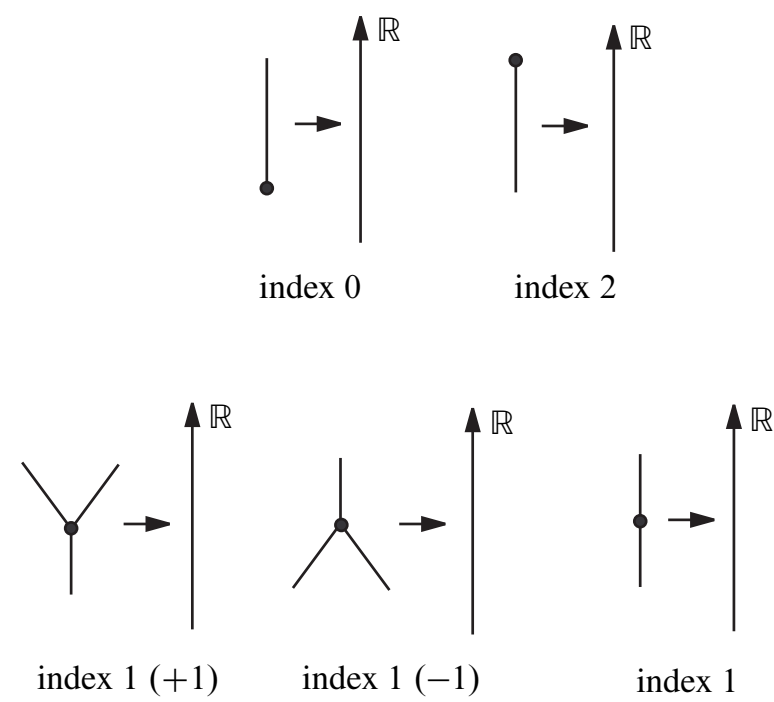

Figure 1: Behavior of $\bar{f}$ around each vertex of the Reeb graph $W_{f}$

To each vertex of degree three of a Reeb graph we associate the sign +1 or -1 as in Figure 1.

For the proof of Theorem 2.4, in [8; 9], certain moves for Reeb functions have been considered (for details, see [8, Figure 3] and [9, Figure 3]). Recall that these moves correspond to the Stein factorizations of cobordisms between Morse functions. We will refer to the types of these moves according to [9, Figure 3] (types (a)-(k)). We call the following types admissible moves for each of the four situations:

(1) oriented cobordism: (a)-(g),

(2) unoriented cobordism: (a)-(k), 
(3) simple oriented cobordism: (a)-(d),

(4) simple unoriented cobordism: (a)-(d), (h), (i).

According to [8; 9], we have only to show that the Reeb function $\bar{f}: W_{f} \rightarrow \mathbb{R}$ associated with an arbitrary stable Morse function $f: M \rightarrow \mathbb{R}$ on a closed (orientable) surface can be deformed to a standard form (see [8, Figure 5] and [9, Figures 4 and 5]) by a finite iteration of admissible moves.

Let us first apply the move (a) to each edge of $W_{f}$ so that $\bar{f}$ decomposes into a disjoint union of four elementary functions as depicted in Figure 2. Note that if $M$ is orientable, then the piece as in Figure 2 (4) does not appear.

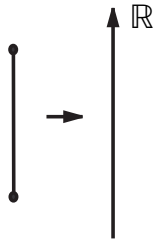

(1)

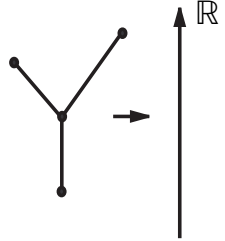

(2)

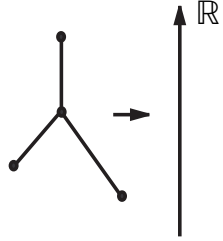

(3)

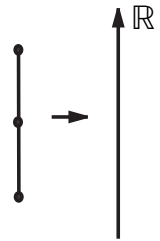

(4)

Figure 2: Four elementary functions

Suppose that $M$ is orientable. If there is a pair of pieces (2) and (3), then by the moves (a) and (c) (or (a) and (b)) we can replace it by one or two pieces of type (1). Furthermore, by the move (d), we may assume that a piece as in (1) does not appear. Therefore, we end up with an empty graph, or a disjoint union of several pieces of type (2) (or a disjoint union of several pieces of type (3)). Then by the move (a), we get a standard form.

Suppose now that $M$ is nonorientable. If there is a pair of pieces of type (4), then we can replace it with a piece (1) by the moves (a) and (i). Then the rest of the argument is the same.

This completes the proof of Theorem 2.4.

It is easy to observe that in the above proof, we have only used admissible moves for simple cobordisms, namely (a)-(d) and (i). Therefore, the same argument can be applied to prove Theorem 2.7 as well.

Remark 3.2 In [8; 9], the moves (e), (f), (g), (j) and (k) were used for the proof, and the same argument cannot be applied to the situation of simple cobordisms. 
In Theorem 2.4 (1) and Theorem 2.7 (1), an isomorphism is given by the map which associates to each cobordism class of a Morse function to the sum of the indices \pm 1 over all vertices of degree three of the associated Reeb function. This sum is equal to the difference between the numbers of local maxima and local minima. In the unoriented cases (Theorem 2.4 (2) and Theorem 2.7 (2)), an isomorphism is constructed by combining a similar map into $\mathbb{Z}$ and the map which associates to each cobordism class of a Morse function to the parity of the number of degree two vertices of the associated Reeb graph. Note that this parity coincides with the parity of the Euler characteristic of the source surface [25, Corollary 2.4].

\section{Universal complex of singular fibers}

In [25], a theory of singular fibers of differentiable maps has been developed. The author has introduced the notion of a universal complex of singular fibers and has shown that certain cohomology classes of a universal complex give rise to cobordism invariants of singular maps. In this section, we show that the isomorphisms in Theorems 2.4 and 2.7 can be given by certain cohomology classes of universal complexes of singular fibers. This will give explicit examples showing the effectiveness of the theory of singular fibers developed in [25].

For the terminologies used in this and the following sections, we refer the reader to [25] and also to $[10 ; 17 ; 28]$.

Let us consider proper $C^{\infty}$ stable maps of 3-manifolds into surfaces. (Recall that for nice dimensions, a proper smooth map is $C^{\infty}$ stable if and only if it is $C^{0}$ stable. See [1].) Then we have the list of $C^{\infty}$ (or $C^{0}$ ) equivalence classes of singular fibers of such maps as in Figure 3. (For the definition of the $C^{\infty}$ or $C^{0}$ equivalence relation for singular fibers, see [25, Chapter 1]. This can be regarded as the $C^{\infty}$ or $C^{0}$ right-left equivalence for map germs along the inverse image of a point.) In fact, every singular fiber of such a map is $C^{\infty}$ (or $C^{0}$ ) equivalent to the disjoint union of one of the fibers as in Figure 3 and a finite number of copies of a fiber of the trivial circle bundle. For details, see [25].

Note that in Figure 3, $\kappa$ denotes the codimension of the set of points in the target whose corresponding fibers are equivalent to the relevant one. Furthermore, $\widetilde{\mathrm{I}}^{*}$ and $\widetilde{\mathrm{II}}^{*}$ mean the name of the corresponding singular fiber, and "/" is used only for separating the figures. The equivalence class of fibers of codimension zero corresponds to the class of regular fibers and is unique. We denote this codimension zero equivalence class by $\tilde{\mathbf{0}}$. We note that the fiber $\widetilde{\text { II }}^{a}$ corresponds to a cusp singular point defined as follows. Let $M$ be a manifold of dimension $n \geq 2$ and $f: M \rightarrow N$ a smooth map into a surface 


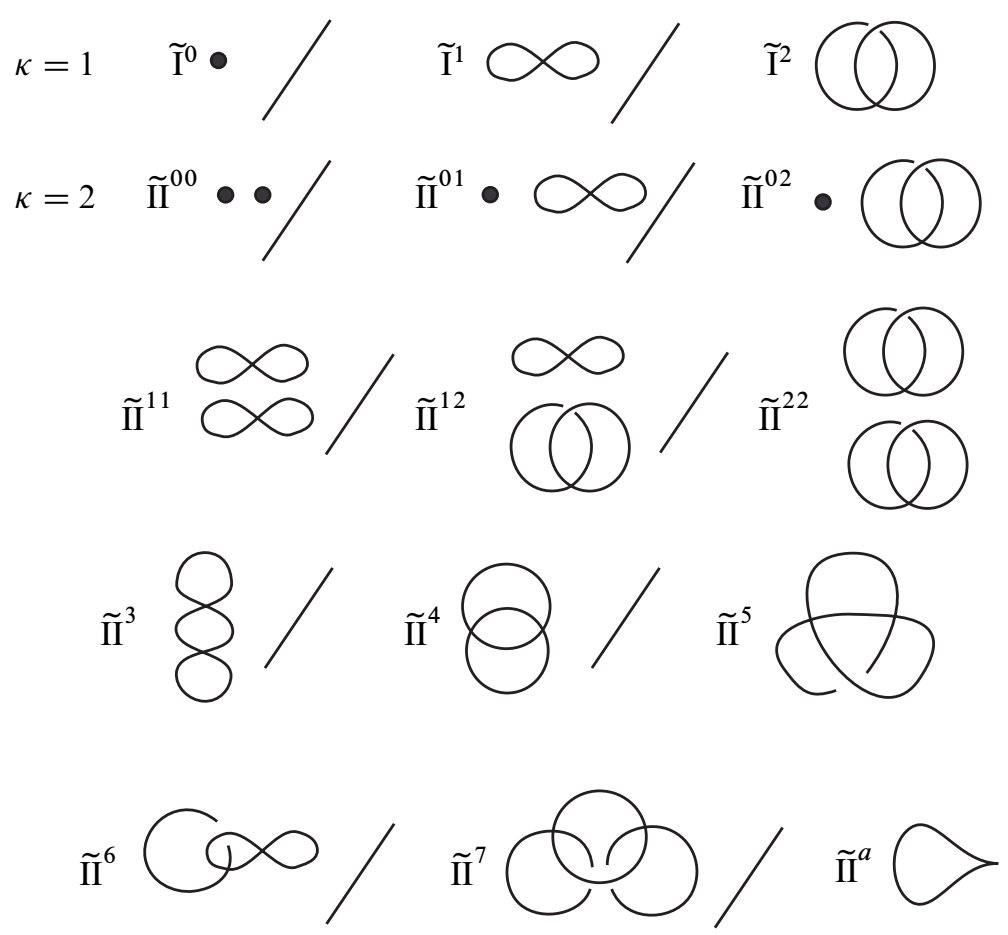

Figure 3: List of singular fibers of proper $C^{\infty}$ stable maps of 3-manifolds into surfaces

$N$. A singular point $x \in S(f)$ of $f$ is called a cusp singular point (or a cusp point) if there exist local coordinates $\left(x_{1}, x_{2}, \ldots, x_{n}\right)$ around $x$ and $\left(y_{1}, y_{2}\right)$ around $f(x)$ such that $f$ has the form

$$
y_{i} \circ f=\left\{\begin{array}{l}
x_{1}, \quad i=1, \\
x_{1} x_{2}+x_{2}^{3} \pm x_{3}^{2} \pm \cdots \pm x_{n}^{2}, \quad i=2 .
\end{array}\right.
$$

If the source 3-manifold is orientable, then the singular fibers of types $\widetilde{\mathrm{I}}^{2}, \widetilde{\mathrm{II}}^{02}, \widetilde{\mathrm{II}}^{12}$, $\widetilde{\mathrm{II}}^{22}, \widetilde{\mathrm{II}}^{5}, \widetilde{\mathrm{II}}^{6}$ and $\widetilde{\mathrm{II}}^{7}$ do not appear.

Note also that the list of $C^{\infty}$ (or $C^{0}$ ) equivalence classes of singular fibers of proper stable Morse functions on surfaces is nothing but those appearing in Figure 3 with $\kappa=1$.

Let $\varrho_{n, n-1}^{0}$ (2) be the $C^{0}$ equivalence relation modulo two circle components for fibers of proper $C^{0}$ stable maps of $n$-dimensional manifolds into $(n-1)$-dimensional manifolds which are Thom maps. (For details, see [25, p. 84]. Roughly speaking, two 
fibers are equivalent with respect to $\varrho_{n, n-1}^{0}$ (2) if one is $C^{0}$ equivalent to the other one after adding an even number of regular circle components.) For a $C^{0}$ equivalence class $\widetilde{\mathfrak{F}}$ of singular fibers, we denote by $\widetilde{\mathfrak{F}}_{\text {o }}$ (or $\widetilde{\mathfrak{F}}_{\text {e }}$ ) the equivalence class with respect to $\varrho_{n, n-1}^{0}(2)$ containing a singular fiber of type $\widetilde{\mathfrak{F}}$ whose total number of components is odd (resp. even).

Let us consider those equivalence classes which are (strongly) co-orientable in the sense of [25, Definition 10.5]. (Roughly speaking, an equivalence class $\widetilde{\mathfrak{F}}_{*}$ is strongly co-orientable if for a given stable map and a point $q$ in the target whose fiber belongs to $\widetilde{\mathfrak{F}}_{*}$, any local homeomorphism around $q$ preserving the adjacent equivalence classes preserves the orientation of the normal direction to the submanifold corresponding to $\widetilde{\mathfrak{F}}_{*}$.) Then we easily get the following for $n=3$.

Lemma 4.1 Those equivalence classes with respect to $\varrho_{3,2}^{0}(2)$ which are strongly co-orientable are $\widetilde{\mathbf{0}}_{*}, \widetilde{\mathrm{I}}_{*}^{0}, \widetilde{\mathrm{I}}_{*}^{1}, \widetilde{\mathrm{I}}_{*}^{01}$ and $\widetilde{\mathrm{II}}_{*}^{a}$, where $*=\mathrm{o}$ and $\mathrm{e}$. The other equivalence classes are not strongly co-orientable.

The above lemma can be proved by observing the degenerations of fibers like those depicted in [25, Figs. 3.5-3.8].

Remark 4.2 If we consider $\varrho_{3,2}^{0}(1)$ ( $C^{0}$ equivalence modulo regular components) instead of $\varrho_{3,2}^{0}(2)$, then no strongly co-orientable equivalence class appears. For this reason, we have chosen the $C^{0}$ equivalence modulo two circle components.

Let $\tau$ be the set of singularity types corresponding to a regular point or a fold point. A smooth map between manifolds is called a $\tau$-map if all of its singularities lie in $\tau$. In other words, a smooth map is a $\tau$-map if and only if it is a fold map in the sense of Section 2. Let us denote by $\tau^{0}(n, p)$ (or $\left.\tau^{0}(n, p)^{\text {ori }}\right)$ the set of all $C^{0}$-equivalence classes of fibers for proper $C^{0}$ stable $\tau$-maps of (orientable) $n-$ dimensional manifolds into $p$-dimensional manifolds which are Thom maps (for details, see [25]). Furthermore, let us denote by $\sigma^{0}(n, p)$ (or $\left.\sigma^{0}(n, p)^{\text {ori }}\right)$ the set of all $C^{0}$-equivalence classes of fibers for proper $C^{0}$ stable simple $\tau$-maps of (orientable) $n$-dimensional manifolds into $p$-dimensional manifolds which are Thom maps. Let

$$
\begin{array}{ll}
\mathcal{C} \mathcal{O}^{*}\left(\tau^{0}(n, n-1), \varrho_{n, n-1}^{0}(2)\right), & \mathcal{C} \mathcal{O}^{*}\left(\tau^{0}(n, n-1)^{\text {ori }}, \varrho_{n, n-1}^{0}(2)\right), \\
\mathcal{C} \mathcal{O}^{*}\left(\sigma^{0}(n, n-1), \varrho_{n, n-1}^{0}(2)\right) & \text { and } \quad \mathcal{C O}^{*}\left(\sigma^{0}(n, n-1)^{\text {ori }}, \varrho_{n, n-1}^{0}(2)\right)
\end{array}
$$


be the universal complexes of co-orientable singular fibers for the respective classes of maps with respect to the $C^{0}$ equivalence modulo two circle components ${ }^{1}$. Note that these complexes are defined over the integers $\mathbb{Z}$.

Then by Lemma 4.1, we see that the following equivalence classes constitute a basis of the $\kappa$-dimensional cochain group for all the four cochain complexes in (4-1) with $n=3$, where $*=\mathrm{o}$ and $\mathrm{e}$ :

$$
\tilde{\mathbf{0}}_{*} \quad(\kappa=0), \quad \widetilde{\mathrm{I}}_{*}^{0}, \widetilde{\mathrm{I}}_{*}^{1} \quad(\kappa=1), \quad \widetilde{\mathrm{II}}_{*}^{01} \quad(\kappa=2) .
$$

Note that $\widetilde{\mathrm{II}}_{*}^{a}$ do not appear, since $\tau$-maps have no cusps. Note also that for $n=2$, we have the same bases for $\kappa \leq 1$.

Let us fix a co-orientation for each of the above equivalence classes. We choose the co-orientation for each of the equivalence classes of codimension one such that the co-orientation points from $\widetilde{\mathbf{0}}_{\mathrm{e}}$ to $\widetilde{\mathbf{0}}_{\mathrm{o}}$. Then we see that the coboundary homomorphism is given by the following formulae (for the definition of the coboundary homomorphisms, see [25, Chapters 7 and 8]):

$$
\begin{aligned}
& \delta_{0}\left(\widetilde{\mathbf{0}}_{\mathrm{o}}\right)=\widetilde{\mathrm{I}}_{\mathrm{o}}^{0}+\widetilde{\mathrm{I}}_{\mathrm{e}}^{0}+\widetilde{\mathrm{I}}_{\mathrm{o}}^{1}+\widetilde{\mathrm{I}}_{\mathrm{e}}^{1}, \\
& \delta_{0}\left(\widetilde{\mathbf{0}}_{\mathrm{e}}\right)=-\widetilde{\mathrm{I}}_{\mathrm{o}}^{0}-\widetilde{\mathrm{I}}_{\mathrm{e}}^{0}-\widetilde{\mathrm{I}}_{\mathrm{o}}^{1}-\widetilde{\mathrm{I}}_{\mathrm{e}}^{1}, \\
& \left.\delta_{1} \widetilde{\mathrm{I}}_{\mathrm{o}}^{0}\right)=\widetilde{\mathrm{II}}_{\mathrm{o}}^{01}-\widetilde{\mathrm{II}}_{\mathrm{e}}^{01}, \\
& \left.\delta_{1} \widetilde{\mathrm{I}}_{\mathrm{e}}^{0}\right)=\widetilde{\mathrm{I}}_{\mathrm{o}}^{01}-\widetilde{\mathrm{I}}_{\mathrm{e}}^{01}, \\
& \left.\delta_{1} \widetilde{\mathrm{I}}_{\mathrm{o}}^{1}\right)=-\widetilde{\mathrm{II}}_{\mathrm{o}}^{01}+\widetilde{\mathrm{II}}_{\mathrm{e}}^{01}, \\
& \left.\delta_{1} \widetilde{\mathrm{I}}_{\mathrm{e}}^{1}\right)=-\widetilde{\mathrm{I}}_{\mathrm{o}}^{01}+\widetilde{\mathrm{II}}_{\mathrm{e}}^{01} .
\end{aligned}
$$

In the following, we denote by $[*]$ the (co)homology class represented by the (co)cycle $*$. Then, by a straightforward calculation, we get the following.

Lemma 4.3 For the cohomology groups of all the four cochain complexes in (4-1) with $n=3$, we have

$$
\begin{aligned}
& H^{0} \cong \mathbb{Z} \quad\left(\text { generated by }\left[\tilde{\mathbf{0}}_{\mathrm{o}}+\widetilde{\mathbf{0}}_{\mathrm{e}}\right]\right), \quad \text { and } \\
& H^{1} \cong \mathbb{Z} \oplus \mathbb{Z} \quad\left(\text { generated by } \alpha_{1}=-\left[\tilde{\mathrm{I}}_{\mathrm{o}}^{0}+\widetilde{\mathrm{I}}_{\mathrm{e}}^{1}\right]=\left[\widetilde{\mathrm{I}}_{\mathrm{e}}^{0}+\widetilde{\mathrm{I}}_{\mathrm{o}}^{1}\right], \alpha_{2}=\left[-\widetilde{\mathrm{I}}_{\mathrm{o}}^{0}+\widetilde{\mathrm{I}}_{\mathrm{e}}^{0}\right],\right. \\
& \left.\quad \text { and } \alpha_{3}=\left[\widetilde{\mathrm{I}}_{\mathrm{o}}^{1}-\widetilde{\mathrm{I}}_{\mathrm{e}}^{1}\right] \text { with } 2 \alpha_{1}=\alpha_{2}+\alpha_{3}\right) .
\end{aligned}
$$

\footnotetext{
${ }^{1}$ In [25], these cochain complexes are denoted by using the symbol " $\mathcal{C O}$ " without " *" as superscripts. However, in this paper, we intentionally put the superscripts in order to distinguish them from the corresponding chain complexes introduced in Section 5.
} 
Furthermore, for $n=2$, the same isomorphism holds for $H^{0}$, and for $H^{1}$, we have

$$
\begin{aligned}
& H^{1} \cong \mathbb{Z} \oplus \mathbb{Z} \oplus \mathbb{Z} \quad\left(\text { generated by } \beta_{1}=-\left[\tilde{\mathrm{I}}_{\mathrm{o}}^{0}+\widetilde{\mathrm{I}}_{\mathrm{e}}^{1}\right]=\left[\tilde{\mathrm{I}}_{\mathrm{e}}^{0}+\widetilde{\mathrm{I}}_{\mathrm{o}}^{1}\right], \beta_{2}=\left[\widetilde{\mathrm{I}}_{\mathrm{o}}^{0}\right],\right. \\
& \text { and } \left.\beta_{3}=\left[\widetilde{\mathrm{I}}_{\mathrm{o}}^{1}\right]\right) .
\end{aligned}
$$

Remark 4.4 The above result for $n=3$ appears to be different from [25, Proposition 14.3]. In fact, in [25], opposite co-orientations are used for $\left[\widetilde{\mathrm{I}}_{\mathrm{o}}^{0}\right]$ and $\left[\widetilde{\mathrm{I}}_{\mathrm{e}}^{1}\right]$.

Let

$$
s_{\kappa}^{0 *}: H^{\kappa}\left(\mathcal{C O}^{*}\left(\tau^{0}(3,2), \varrho_{3,2}^{0}(2)\right)\right) \rightarrow H^{\kappa}\left(\mathcal{C O}^{*}\left(\tau^{0}(2,1), \varrho_{2,1}^{0}(2)\right)\right)
$$

etc. be the homomorphism induced by suspension ${ }^{2}$. Then for $\kappa=1$, we have

$$
s_{1}^{0 *} \alpha_{1}=\beta_{1}, s_{1}^{0 *} \alpha_{2}=\beta_{1}-\beta_{2}-\beta_{3} \text { and } s_{1}^{0 *} \alpha_{3}=\beta_{1}+\beta_{2}+\beta_{3} .
$$

In particular, we see that $s_{1}^{0 *}$ is injective and its image is isomorphic to $\mathbb{Z} \oplus \mathbb{Z}$.

Let $f: M \rightarrow \mathbb{R}$ be an arbitrary stable Morse function on a closed surface $M$. We give the orientation to $\mathbb{R}$ which points to the increasing direction. For a $\kappa$-dimensional cohomology class $\alpha$ of the universal complex of co-orientable singular fibers represented by a cocycle $c$, we denote by $\alpha(f) \in H_{1-\kappa}(\mathbb{R} ; \mathbb{Z})$ the homology class ${ }^{3}$ represented by the cycle corresponding to the closure of the set of points in $\mathbb{R}$ whose associated fiber belongs to an equivalence class appearing in $c$ (for details, see [25, Chapter 11]) ${ }^{4}$.

Then by the same argument as in the proof of [25, Lemma 14.1], we see that

$$
s_{1}^{0 *} \alpha_{1}(f)=\beta_{1}(f) \in H_{0}(\mathbb{R} ; \mathbb{Z}) \cong \mathbb{Z}
$$

always vanishes (see also Remark 6.5 of the present paper). Furthermore, we have the following.

Lemma 4.5 For a Morse function $f$ as above, we have

$$
s_{1}^{0 *} \alpha_{2}(f)=-s_{1}^{0 *} \alpha_{3}(f)=\max (f)-\min (f)
$$

under the natural identification $H_{0}(\mathbb{R} ; \mathbb{Z})=\mathbb{Z}$, where $\max (f)$ (or $\min (f)$ ) is the number of local maxima (resp. minima) of the Morse function $f$.

\footnotetext{
${ }^{2}$ In [25], the notation $s_{\kappa *}^{0}$ is used instead of $s_{\kappa}^{0 *}$. However, the latter should have been used, since it corresponds to a pull-back. More details will be explained in Section 5.

${ }^{3}$ For $\kappa=0$ we consider the homology group with closed support.

${ }^{4}$ More precisely, we consider the chain with multiplicity given by the corresponding coefficient in $c$.
} 
Proof Let $c \in \mathbb{R}$ be a value corresponding to a local minimum of $f$. If $f^{-1}(c)$ has an odd (or even) number of components, then it contributes +1 (resp. -1$)$ to $\left\|\widetilde{\mathrm{I}}_{\mathrm{o}}^{0}(f)\right\|$ (resp. $\left\|\widetilde{\mathrm{I}}_{\mathrm{e}}^{0}(f)\right\|$ ), where $\|*\|$ refers to the algebraic number of elements, and for an equivalence class $\widetilde{\mathfrak{F}}, \tilde{\mathfrak{F}}(f)$ denotes the set of points in $\mathbb{R}$ over which lies a singular fiber of type $\widetilde{\mathfrak{F}}$. If $c$ corresponds to a local maximum, then the signs of contribution change in both cases. Therefore, we have the desired conclusion.

Note that by [25], for any 1-dimensional cohomology class $\alpha$ of the universal complex as in (4-1) with $n=3, s_{1}^{0 *} \alpha(f) \in H_{0}(\mathbb{R} ; \mathbb{Z}) \cong \mathbb{Z}$ gives a fold cobordism invariant for stable Morse functions $f$ on closed surfaces. By the proofs of Theorem 2.4 (1) and Theorem 2.7 (1), we see that the maps

$$
\Phi^{S O}: \mathcal{M}^{S O}(2) \rightarrow \mathbb{Z} \quad \text { and } \quad S \Phi^{S O}: \mathcal{S M}^{S O}(2) \rightarrow \mathbb{Z}
$$

which send the cobordism class of a stable Morse function $f$ to $s_{1}^{0 *} \alpha_{2}(f)=\max (f)-$ $\min (f) \in \mathbb{Z}$ are isomorphisms.

In the unoriented case, the corresponding maps do not give isomorphisms according to Theorem 2.4 (2) and Theorem 2.7 (2). In order to get isomorphisms, let us consider the universal complexes of singular fibers

$$
\mathcal{C}^{*}\left(\tau^{0}(n, n-1), \varrho_{n, n-1}^{0}(2)\right) \quad \text { and } \quad \mathcal{C}^{*}\left(\sigma^{0}(n, n-1), \varrho_{n, n-1}^{0}(2)\right)
$$

with coefficients in $\mathbb{Z}_{2}$. (Here again, we put “ $*$ ” as superscripts.)

The coboundary homomorphisms for the case of $\tau^{0}(3,2)$ satisfy the following:

$$
\begin{aligned}
& \delta_{0}\left(\widetilde{\mathbf{0}}_{\mathrm{o}}\right)=\widetilde{\mathrm{I}}_{\mathrm{o}}^{0}+\widetilde{\mathrm{I}}_{\mathrm{e}}^{0}+\widetilde{\mathrm{I}}_{\mathrm{o}}^{1}+\widetilde{\mathrm{I}}_{\mathrm{e}}^{1}, \\
& \delta_{0}\left(\widetilde{\mathbf{0}}_{\mathrm{e}}\right)=\widetilde{\mathrm{I}}_{\mathrm{o}}^{0}+\widetilde{\mathrm{I}}_{\mathrm{e}}^{0}+\widetilde{\mathrm{I}}_{\mathrm{o}}^{1}+\widetilde{\mathrm{I}}_{\mathrm{e}}^{1}, \\
& \delta_{1}\left(\widetilde{\mathrm{I}}_{\mathrm{o}}^{0}\right)=\widetilde{\mathrm{II}}_{\mathrm{o}}^{01}+\widetilde{\mathrm{II}}_{\mathrm{e}}^{01}, \\
& \delta_{1}\left(\widetilde{\mathrm{I}}_{\mathrm{e}}^{0}\right)=\widetilde{\mathrm{II}}_{\mathrm{o}}^{01}+\widetilde{\mathrm{II}}_{\mathrm{e}}^{01}, \\
& \delta_{1}\left(\widetilde{\mathrm{I}}_{\mathrm{o}}^{1}\right)=\widetilde{\mathrm{II}}_{\mathrm{o}}^{01}+\widetilde{\mathrm{II}}_{\mathrm{e}}^{01}, \\
& \left.\delta_{1} \widetilde{\mathrm{I}}_{\mathrm{e}}^{1}\right)=\widetilde{\mathrm{II}}_{\mathrm{o}}^{01}+\widetilde{\mathrm{II}}_{\mathrm{e}}^{01}, \\
& \delta_{1}\left(\widetilde{\mathrm{I}}_{\mathrm{o}}^{2}\right)=\widetilde{\mathrm{II}}_{\mathrm{o}}^{02}+\widetilde{\mathrm{II}}_{\mathrm{e}}^{02}+\widetilde{\mathrm{II}}_{\mathrm{o}}^{12}+\widetilde{\mathrm{II}}_{\mathrm{e}}^{12}+\widetilde{\mathrm{II}}_{\mathrm{o}}^{6}+\widetilde{\mathrm{II}}_{\mathrm{e}}^{6}, \\
& \left.\delta_{1} \widetilde{\mathrm{I}}_{\mathrm{e}}^{2}\right)=\widetilde{\mathrm{II}}_{\mathrm{o}}^{02}+\widetilde{\mathrm{II}}_{\mathrm{e}}^{02}+\widetilde{\mathrm{II}}_{\mathrm{o}}^{12}+\widetilde{\mathrm{II}}_{\mathrm{e}}^{12}+\widetilde{\mathrm{II}}_{\mathrm{o}}^{6}+\widetilde{\mathrm{II}}_{\mathrm{e}}^{6} .
\end{aligned}
$$

For the case of $\sigma^{0}(3,2)$, we obtain the formulae for the coboundary homomorphisms by ignoring $\widetilde{\mathrm{II}}_{*}^{6}$ above. For the cases of $\tau^{0}(2,1)$ and $\sigma^{0}(2,1)$, the same formulae hold for $\delta_{0}$. 
By a straightforward calculation, we get the following.

Lemma 4.6 For the cohomology groups of the cochain complexes in (4-3) with $n=3$, we have

$$
\begin{aligned}
& H^{0} \cong \mathbb{Z}_{2} \quad\left(\text { generated by }\left[\tilde{\mathbf{0}}_{\mathrm{o}}+\widetilde{\mathbf{0}}_{\mathrm{e}}\right]\right), \quad \text { and } \\
& H^{1} \cong \mathbb{Z}_{2} \oplus \mathbb{Z}_{2} \oplus \mathbb{Z}_{2} \quad\left(\text { generated by } \widehat{\alpha}_{1}=\left[\widetilde{\mathrm{I}}_{\mathrm{o}}^{0}+\widetilde{\mathrm{I}}_{\mathrm{e}}^{1}\right]=\left[\widetilde{\mathrm{I}}_{\mathrm{e}}^{0}+\widetilde{\mathrm{I}}_{\mathrm{o}}^{1}\right],\right. \\
& \left.\quad \widehat{\alpha}_{2}=\left[\widetilde{\mathrm{I}}_{\mathrm{o}}^{0}+\widetilde{\mathrm{I}}_{\mathrm{e}}^{0}\right]=\left[\tilde{\mathrm{I}}_{\mathrm{o}}^{1}+\widetilde{\mathrm{I}}_{\mathrm{e}}^{1}\right] \text { and } \widehat{\alpha}_{3}=\left[\tilde{\mathrm{I}}_{\mathrm{o}}^{2}+\widetilde{\mathrm{I}}_{\mathrm{e}}^{2}\right]\right) .
\end{aligned}
$$

Furthermore, for $n=2$, the same isomorphism holds for $H^{0}$, and for $H^{1}$, we have

$$
\begin{aligned}
& H^{1} \cong \mathbb{Z}_{2} \oplus \mathbb{Z}_{2} \oplus \mathbb{Z}_{2} \oplus \mathbb{Z}_{2} \oplus \mathbb{Z}_{2} \quad\left(\text { generated by } \hat{\beta}_{1}=\left[\tilde{\mathrm{I}}_{\mathrm{o}}^{0}+\widetilde{\mathrm{I}}_{\mathrm{e}}^{1}\right]=\left[\widetilde{\mathrm{I}}_{\mathrm{e}}^{0}+\widetilde{\mathrm{I}}_{\mathrm{o}}^{1}\right],\right. \\
& \left.\hat{\beta}_{2}=\left[\widetilde{\mathrm{I}}_{\mathrm{o}}^{0}\right], \hat{\beta}_{3}=\left[\widetilde{\mathrm{I}}_{\mathrm{o}}^{1}\right], \hat{\beta}_{4}=\left[\widetilde{\mathrm{I}}_{\mathrm{o}}^{2}\right] \text { and } \hat{\beta}_{5}=\left[\widetilde{\mathrm{I}}_{\mathrm{e}}^{2}\right]\right) .
\end{aligned}
$$

We can also describe the homomorphisms induced by suspension with respect to the above generators.

Let $f: M \rightarrow \mathbb{R}$ be a stable Morse function on a closed surface $M$. Then we see that $s_{1}^{0 *} \hat{\alpha}_{1}(f) \in H_{0}\left(\mathbb{R} ; \mathbb{Z}_{2}\right) \cong \mathbb{Z}_{2}$ always vanishes as before. Furthermore, $s_{1}^{0 *} \hat{\alpha}_{2}(f)$ coincides with $\min (f)+\max (f)$ modulo two. Finally, $s_{1}^{0 *} \hat{\alpha}_{3}(f)$ gives the number of singular fibers of type $\widetilde{\mathrm{I}}^{2}$ of $f$. Therefore, according to the proofs of Theorem 2.4 (2) and Theorem 2.7 (2), we see that the homomorphisms

$$
\Phi: \mathcal{M}(2) \rightarrow \mathbb{Z} \oplus \mathbb{Z}_{2} \quad \text { and } \quad S \Phi: \mathcal{S M}(2) \rightarrow \mathbb{Z} \oplus \mathbb{Z}_{2}
$$

which send the cobordism class of a stable Morse function $f$ to

$$
\left(s_{1}^{0 *} \alpha_{2}(f), s_{1}^{0 *} \widehat{\alpha}_{3}(f)\right)=\left(\max (f)-\min (f),\left|\tilde{\mathrm{I}}^{2}(f)\right|\right) \in \mathbb{Z} \oplus \mathbb{Z}_{2}
$$

are isomorphisms, where $|*|$ denotes the number of elements modulo two.

Note that by [25, Corollary 2.4], $\left|\widetilde{\mathbf{I}}^{2}(f)\right| \in \mathbb{Z}_{2}$ coincides with the parity of the Euler characteristic $\chi(M)$ of the surface $M$.

As the above observations show, the cohomology classes of universal complexes of singular fibers can give complete cobordism invariants for singular maps.

\section{Universal homology complex of singular fibers}

In the previous section, we have seen that cohomology classes of the universal complexes of singular fibers give rise to complete cobordism invariants in our situations. In order to construct such invariants in the unoriented case, we had to combine the universal 
complex of co-orientable singular fibers and that of usual singular fibers which are not necessarily co-orientable.

In [10], Kazarian introduced the notion of a universal homology complex of singularities, which combines the universal cohomology complex of co-orientable singularities and that of usual (not necessarily co-orientable) singularities, and which is constructed by reversing the arrows. In this section, we will pursue the same procedure in our situation of singular fibers.

Let us consider the case of proper $C^{0}$ stable fold maps of (possibly nonorientable) $n$-dimensional manifolds into $(n-1)$-dimensional manifolds. (The case of simple maps or that of maps of oriented manifolds can be treated similarly.) Let

$$
\mathcal{C}_{*}\left(\tau^{0}(n, n-1), \varrho_{n, n-1}^{0}(2)\right)
$$

be the chain complex defined as follows. For each $\kappa$, the $\kappa$-dimensional chain group, denoted by $C_{\kappa}\left(\tau^{0}(n, n-1), \varrho_{n, n-1}^{0}(2)\right)$, is the direct sum, over all equivalence classes of singular fibers of codimension $\kappa$ with respect to $\varrho_{n, n-1}^{0}(2)$, of the groups $\mathbb{Z}$ for co-orientable classes and the groups $\mathbb{Z}_{2}$ for non co-orientable classes, and we denote the generators by using the same symbols for the corresponding equivalence classes of singular fibers.

Let $\mathfrak{F}$ and $\mathfrak{G}$ be two equivalence classes of singular fibers such that $\kappa(\mathfrak{F})=\kappa(\mathfrak{G})+1$, where $\kappa$ denotes the codimension. Let $f: M \rightarrow N$ be a proper $C^{0}$ stable fold map of an $n$-dimensional manifold into an $(n-1)$-dimensional manifold which is a Thom map. Let us denote by $\mathfrak{F}(f)$ (or $\mathfrak{G}(f)$ ) the set of points in $N$ over which lies a singular fiber of type $\mathfrak{F}$ (resp. $\mathfrak{G})$. Note that $\mathfrak{F}(f)$ and $\mathfrak{G}(f)$ are submanifolds of $N$ of codimensions $\kappa(\mathfrak{F})$ and $\kappa(\mathfrak{G})$ respectively. Let us consider a point $q \in \mathfrak{F}(f)$ and a small disk $D_{q}$ of dimension $\kappa(\mathfrak{F})$ centered at $q$ which intersects $\mathfrak{F}(f)$ transversely exactly at $q$. Then $\mathfrak{G}(f)$ cuts $D_{q}$ in a finite set of curves. If $\mathfrak{G}$ is not co-orientable, then we define $[\mathfrak{G}: \mathfrak{F}] \in \mathbb{Z}_{2}$ as the parity of the number of these curves. If $\mathfrak{G}$ is co-orientable, then the chosen co-orientation of $\mathfrak{G}$ together with the chosen orientation of $D_{q}$ allows us to define a sign for each curve. We define $[\mathfrak{G}: \mathfrak{F}] \in \mathbb{Z}$ as the algebraic number of these curves, counted with signs. Note that if $\mathfrak{G}$ is co-orientable and $\mathfrak{F}$ is not co-orientable, then we always have $[\mathfrak{G}: \mathfrak{F}]=0$. Note also that the incidence coefficient $[\mathfrak{G}: \mathfrak{F}]$ thus defined does not depend on the choice of $q$ etc. and is well-defined for all the above cases.

Now the boundary homomorphism

$$
\partial_{\kappa}: C_{\kappa}\left(\tau^{0}(n, n-1), \varrho_{n, n-1}^{0}(2)\right) \rightarrow C_{\kappa-1}\left(\tau^{0}(n, n-1), \varrho_{n, n-1}^{0}(2)\right)
$$


is defined by the formula

$$
\partial_{\kappa}(\mathfrak{F})=\sum_{\kappa(\mathfrak{G})=\kappa(\mathfrak{F})-1}[\mathfrak{G}: \mathfrak{F}] \mathfrak{G}
$$

for the generators $\mathfrak{F}$ of $C_{\kappa}\left(\tau^{0}(n, n-1), \varrho_{n, n-1}^{0}(2)\right)$. Note that this is a well-defined homomorphism.

It is easy to check that $\partial_{\kappa-1} \circ \partial_{\kappa}=0$ as in $[25 ; 28]$. The chain complex

$$
\mathcal{C}_{*}\left(\tau^{0}(n, n-1), \varrho_{n, n-1}^{0}(2)\right)=\left(C_{\kappa}\left(\tau^{0}(n, n-1), \varrho_{n, n-1}^{0}(2)\right), \partial_{\kappa}\right)_{\kappa}
$$

thus constructed is called the universal homology complex of singular fibers for $C^{0}$ stable fold maps of $n$-dimensional manifolds into $(n-1)$-dimensional manifolds.

Remark 5.1 In the definition of the universal complex given in [25], we have formally allowed infinite sums as elements of the cochain groups. However, for the universal homology complex that we have defined here, we consider the direct sum of some copies of $\mathbb{Z}$ and $\mathbb{Z}_{2}$, and we do not allow infinite sums. Therefore, the boundary homomorphism is well-defined.

As in [10], we can check that the universal cochain complex of singular fibers

$$
\mathcal{C}^{*}\left(\tau^{0}(n, n-1), \varrho_{n, n-1}^{0}(2)\right)
$$

and the universal cochain complex of co-orientable singular fibers

$$
\mathcal{C} \mathcal{O}^{*}\left(\tau^{0}(n, n-1), \varrho_{n, n-1}^{0}(2)\right)
$$

as defined in [25] are isomorphic to

$$
\operatorname{Hom}\left(\mathcal{C}_{*}\left(\tau^{0}(n, n-1), \varrho_{n, n-1}^{0}(2)\right), \mathbb{Z}_{2}\right)
$$

and

$$
\operatorname{Hom}\left(\mathcal{C}_{*}\left(\tau^{0}(n, n-1), \varrho_{n, n-1}^{0}(2)\right), \mathbb{Z}\right)
$$

respectively. In this sense, the universal homology complex (5-1) unifies the universal complex of usual singular fibers with coefficients in $\mathbb{Z}_{2}$ and that of co-orientable ones with coefficients in $\mathbb{Z}$.

Let us proceed to the explicit calculation in the case of $n=3$. The generators of $C_{\kappa}\left(\tau^{0}(3,2), \varrho_{3,2}^{0}(2)\right)$ are as given in Table 1 (see also Figure 3 ).

Algebraic $8 \mathcal{G}$ Geometric Topology, Volume 6 (2006) 
The boundary homomorphisms are given as follows.

$$
\begin{aligned}
& \widetilde{\mathrm{II}}_{\mathrm{o}}^{01} \mapsto \widetilde{\mathrm{I}}_{\mathrm{o}}^{0}+\widetilde{\mathrm{I}}_{\mathrm{e}}^{0}-\widetilde{\mathrm{I}}_{\mathrm{o}}^{1}-\widetilde{\mathrm{I}}_{\mathrm{e}}^{1}, \\
& \widetilde{\mathrm{I}}_{\mathrm{e}}^{01} \mapsto-\widetilde{\mathrm{I}}_{\mathrm{o}}^{0}-\widetilde{\mathrm{I}}_{\mathrm{e}}^{0}+\widetilde{\mathrm{I}}_{\mathrm{o}}^{1}+\widetilde{\mathrm{I}}_{\mathrm{e}}^{1}, \\
& \widetilde{\mathrm{II}}_{\mathrm{o}}^{00}, \widetilde{\mathrm{II}}_{\mathrm{e}}^{00}, \widetilde{\mathrm{II}}_{\mathrm{o}}^{11}, \widetilde{\mathrm{II}}_{\mathrm{e}}^{11}, \widetilde{\mathrm{II}}_{\mathrm{o}}^{22}, \widetilde{\mathrm{II}}_{\mathrm{e}}^{22}, \widetilde{\mathrm{II}}_{\mathrm{o}}^{3}, \widetilde{\mathrm{II}}_{\mathrm{e}}^{3}, \widetilde{\mathrm{II}}_{\mathrm{o}}^{4}, \widetilde{\mathrm{II}}_{\mathrm{e}}^{4}, \widetilde{\mathrm{II}}_{\mathrm{o}}^{5}, \widetilde{\mathrm{I}}_{\mathrm{e}}^{5}, \widetilde{\mathrm{II}}_{\mathrm{o}}^{7}, \widetilde{\mathrm{I}}_{\mathrm{e}}^{7} \mapsto 0, \\
& \widetilde{\mathrm{II}}_{\mathrm{o}}^{02}, \widetilde{\mathrm{II}}_{\mathrm{e}}^{02}, \widetilde{\mathrm{I}}_{\mathrm{o}}^{12}, \widetilde{\mathrm{II}}_{\mathrm{e}}^{12}, \widetilde{\mathrm{II}}_{\mathrm{o}}^{6}, \widetilde{\mathrm{II}}_{\mathrm{e}}^{6} \mapsto \widetilde{\mathrm{I}}_{\mathrm{o}}^{2}+\widetilde{\mathrm{I}}_{\mathrm{e}}^{2}, \\
& \widetilde{\mathrm{I}}_{\mathrm{o}}^{0}, \widetilde{\mathrm{I}}_{\mathrm{e}}^{0}, \widetilde{\mathrm{I}}_{\mathrm{o}}^{1}, \widetilde{\mathrm{I}}_{\mathrm{e}}^{1} \mapsto \widetilde{\mathbf{0}}_{\mathrm{o}}-\widetilde{\mathbf{0}}_{\mathrm{e}}, \\
& \widetilde{\mathrm{I}}_{\mathrm{o}}^{2}, \widetilde{\mathrm{I}}_{\mathrm{e}}^{2} \mapsto 0 .
\end{aligned}
$$

Then a straightforward calculation shows the following.

Lemma 5.2 For the homology groups of the chain complex

$$
\mathcal{C}_{*}\left(\tau^{0}(3,2), \varrho_{3,2}^{0}(2)\right)
$$

we have

$$
\begin{aligned}
& H_{0} \cong \mathbb{Z} \quad\left(\text { generated by }\left[\widetilde{\mathbf{0}}_{\mathrm{o}}\right]=\left[\tilde{\mathbf{0}}_{\mathrm{e}}\right]\right), \quad \text { and } \\
& H_{1} \cong \mathbb{Z} \oplus \mathbb{Z} \oplus \mathbb{Z}_{2} \quad\left(\text { generated by } \widetilde{\alpha}_{1}=\left[\widetilde{\mathrm{I}}_{\mathrm{o}}^{0}-\widetilde{\mathrm{I}}_{\mathrm{e}}^{1}\right]=\left[\widetilde{\mathrm{I}}_{\mathrm{o}}^{1}-\widetilde{\mathrm{I}}_{\mathrm{e}}^{0}\right],\right. \\
& \left.\widetilde{\alpha}_{2}=\left[-\widetilde{\mathrm{I}}_{\mathrm{o}}^{0}+\widetilde{\mathrm{I}}_{\mathrm{e}}^{0}\right] \text { and } \widetilde{\alpha}_{3}=\left[\widetilde{\mathrm{I}}_{\mathrm{o}}^{2}\right]=\left[\widetilde{\mathrm{I}}_{\mathrm{e}}^{2}\right]\right) .
\end{aligned}
$$

Note that for $H_{1}$, we can replace $\widetilde{\alpha}_{2}$ by

$$
\widetilde{\alpha}_{2}^{\prime}=\left[-\widetilde{\mathrm{I}}_{\mathrm{o}}^{1}+\widetilde{\mathrm{I}}_{\mathrm{e}}^{1}\right]
$$

since we have the relation $-2 \widetilde{\alpha}_{1}=\widetilde{\alpha}_{2}+\widetilde{\alpha}_{2}^{\prime}$.

In order to consider the hypercohomologies, in the sense of [10], of the universal homology complex constructed above, let us consider a free approximation $\mathcal{F}$ of

$$
\mathcal{V}=\mathcal{C}_{*}\left(\tau^{0}(3,2), \varrho_{3,2}^{0}(2)\right)
$$

Table 1: Generators of $C_{\kappa}\left(\tau^{0}(3,2), \varrho_{3,2}^{0}(2)\right)$

\begin{tabular}{|c||c|c|}
\hline$\kappa$ & $\mathbb{Z}$ & $\mathbb{Z}_{2}$ \\
\hline \hline 0 & $\widetilde{\mathbf{0}}_{\mathrm{o}}, \widetilde{\mathbf{0}}_{\mathrm{e}}$ & \\
\hline 1 & $\widetilde{\mathrm{I}}_{\mathrm{o}}^{0}, \widetilde{\mathrm{I}}_{\mathrm{e}}^{0}, \widetilde{\mathrm{I}}_{\mathrm{o}}^{1}, \widetilde{\mathrm{I}}_{\mathrm{e}}^{1}$ & $\widetilde{\mathrm{I}}_{\mathrm{o}}^{2}, \widetilde{\mathrm{I}}_{\mathrm{e}}^{2}$ \\
\hline 2 & $\widetilde{\mathrm{II}}_{\mathrm{o}}^{01}, \widetilde{\mathrm{II}}_{\mathrm{e}}^{01}$ & $\widetilde{\mathrm{II}}_{\mathrm{o}}^{00}, \widetilde{\mathrm{II}}_{\mathrm{e}}^{00}, \widetilde{\mathrm{II}}_{\mathrm{o}}^{11}, \widetilde{\mathrm{I}}_{\mathrm{e}}^{11}, \widetilde{\mathrm{II}}_{\mathrm{o}}^{02}, \widetilde{\mathrm{I}}_{\mathrm{e}}^{02}, \widetilde{\mathrm{II}}_{\mathrm{o}}^{12}, \widetilde{\mathrm{I}}_{\mathrm{e}}^{12}, \widetilde{\mathrm{I}}_{\mathrm{o}}^{22}, \widetilde{\mathrm{I}}_{\mathrm{e}}^{22}$, \\
& & $\widetilde{\mathrm{II}}_{\mathrm{o}}^{3}, \widetilde{\mathrm{II}}_{\mathrm{e}}^{3}, \widetilde{\mathrm{II}}_{\mathrm{o}}^{4}, \widetilde{\mathrm{II}}_{\mathrm{e}}^{4}, \widetilde{\mathrm{II}}_{\mathrm{o}}^{5}, \widetilde{\mathrm{II}}_{\mathrm{e}}^{5}, \widetilde{\mathrm{II}}_{\mathrm{o}}^{6}, \widetilde{\mathrm{II}}_{\mathrm{e}}^{6}, \widetilde{\mathrm{II}}_{\mathrm{o}}^{7}, \widetilde{\mathrm{II}}_{\mathrm{e}}^{7}$ \\
\hline
\end{tabular}


(for the definition of a free approximation, see [26, Chapter 5, Section 2] or [10]). For each $\kappa$, let us denote the $\kappa$-dimensional cochain group of $\mathcal{F}$ by $F_{\kappa}$. Then the generators of the free abelian group $F_{\kappa}, \kappa=0,1,2$, are given by the elements corresponding to those of $\mathcal{V}$, except that we need to add one generator $A$ to $F_{2}$. We denote the corresponding generators by the same symbols as in Table 1 . The boundary homomorphism $\partial_{\kappa}: F_{\kappa} \rightarrow F_{\kappa-1}$ is given by the same formulae as in (5-2) and by

$$
\partial_{2}(A)=2 \widetilde{\mathrm{I}}_{\mathrm{o}}^{2} .
$$

Furthermore, the epimorphism $\lambda: \mathcal{F} \rightarrow \mathcal{V}$ is naturally defined by the obvious correspondence together with $\lambda(A)=0$. It is straightforward to check that $\lambda$ is a chain map and the induced homomorphism $\lambda_{*}: H_{*}(\mathcal{F} ; \mathbb{Z}) \rightarrow H_{*}(\mathcal{V} ; \mathbb{Z})$ is an isomorphism.

For an abelian group $G$, the hypercohomology $\mathbb{\boxplus}^{*}(\mathcal{V} ; G)$ of $\mathcal{V}$ with coefficients in $G$ is, by definition, $H^{*}(\mathcal{F} ; G)$. This is well-defined and depends only on $\mathcal{V}$ and $G$ (for details, see [26]).

Recall the canonical isomorphisms:

$$
\begin{aligned}
\mathcal{C}^{*}\left(\tau^{0}(3,2), \varrho_{3,2}^{0}(2)\right) & \cong \operatorname{Hom}\left(\mathcal{V}, \mathbb{Z}_{2}\right) \text { and } \\
\mathcal{C} \mathcal{O}^{*}\left(\tau^{0}(3,2), \varrho_{3,2}^{0}(2)\right) & \cong \operatorname{Hom}(\mathcal{V}, \mathbb{Z}) .
\end{aligned}
$$

Then a straightforward calculation shows the following.

Lemma 5.3 The following homomorphisms induced by $\lambda$ are both isomorphisms for $\kappa=0,1$ :

$$
\begin{aligned}
H^{\kappa}\left(\mathcal{C}^{*}\left(\tau^{0}(3,2), \varrho_{3,2}^{0}(2)\right)=H^{\kappa}\left(\mathcal{V} ; \mathbb{Z}_{2}\right)\right. & \\
\rightarrow & H^{\kappa}\left(\mathcal{F} ; \mathbb{Z}_{2}\right)=\mathbb{H}^{\kappa}\left(\mathcal{C}_{*}\left(\tau^{0}(3,2), \varrho_{3,2}^{0}(2)\right) ; \mathbb{Z}_{2}\right), \\
H^{\kappa}\left(\mathcal{C} \mathcal{O}^{*}\left(\tau^{0}(3,2), \varrho_{3,2}^{0}(2)\right)=H^{\kappa}(\mathcal{V} ; \mathbb{Z})\right. & \\
& \rightarrow H^{\kappa}(\mathcal{F} ; \mathbb{Z})=\mathbb{H}^{\kappa}\left(\mathcal{C}_{*}\left(\tau^{0}(3,2), \varrho_{3,2}^{0}(2)\right) ; \mathbb{Z}\right) .
\end{aligned}
$$

Remark 5.4 As the above lemma shows, for $n=3$ the homomorphisms $\lambda^{*}$ are isomorphisms. However, for $n>3$, we do not know if this is true or not.

Let $f: M \rightarrow N$ be a proper $C^{0}$ stable $\tau$-map of an $n$-dimensional manifold into an $(n-1)$-dimensional manifold which is a Thom map. Then by the same argument as in [10, Section 5], we can define a natural homomorphism

$$
\tilde{\varphi}_{f}^{*}: \mathbb{T}^{*}(\mathcal{V}(n, n-1) ; G) \rightarrow H^{*}(N ; G)
$$


in such a way that for $G=\mathbb{Z}$ and $\mathbb{Z}_{2}$, we have

$$
\varphi_{f}^{*}=\tilde{\varphi}_{f}^{*} \circ \lambda^{*}: H^{*}(\mathcal{V}(n, n-1) ; G) \rightarrow H^{*}(N ; G),
$$

where $\varphi_{f}^{*}$ refers to the homomorphism induced by $f$ in the sense of [25, Chapter 11] and

$$
\mathcal{V}(n, n-1)=\mathcal{C}_{*}\left(\tau^{0}(n, n-1), \varrho_{n, n-1}^{0}(2)\right) .
$$

(We have added "*” as superscript for $\varphi_{f}^{*}$, which will be necessary in the following argument.) Note also that we can define the natural homomorphism

$$
\varphi_{f *}=\tilde{\varphi}_{f *}: H_{*}(N ; G) \rightarrow \mathbb{H}_{*}(\mathcal{V}(n, n-1) ; G)=H_{*}(\mathcal{V}(n, n-1) ; G)
$$

for any abelian group $G$. (In fact, $\varphi_{f}$ and $\tilde{\varphi}_{f}$ are defined on the chain level.)

Let us show that the homomorphism $\widetilde{\varphi}_{f}^{*}$ induced by $f$ defines a $\tau$-cobordism invariant of $f$.

By virtue of the uniqueness of the lift (up to chain homotopy) for free approximations in the sense of [26, Chapter 2, Section 2, Lemma 13] and [10, Proposition 2.2], we can define the suspension homomorphism for free approximations of the universal homology complexes of singular fibers. More precisely, we have a natural chain map

$$
s: \mathcal{C}_{*}\left(\tau^{0}(n, n-1), \varrho_{n, n-1}^{0}(2)\right) \rightarrow \mathcal{C}_{*}\left(\tau^{0}(n+1, n), \varrho_{n+1, n}^{0}(2)\right)
$$

induced by suspension. (Recall that the suspension of a map $f: M \rightarrow N$ refers to the map $f \times \operatorname{id}_{\mathbb{R}}: M \times \mathbb{R} \rightarrow N \times \mathbb{R}$ and this naturally induces the notion of a suspension for singular fibers. For details, see [25, Definition 8.4].) Then we have a chain map $\tilde{s}: \mathcal{F}(n, n-1) \rightarrow \mathcal{F}(n+1, n)$, unique up to chain homotopy, which makes the following diagram commutative:

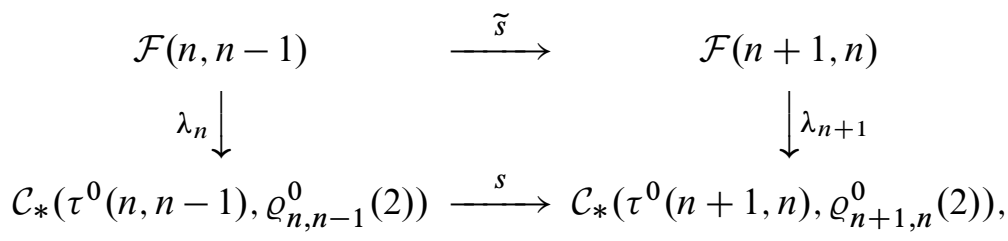

where $\mathcal{F}(m, m-1)$ denotes a free approximation of $\mathcal{C}_{*}\left(\tau^{0}(m, m-1), \varrho_{m, m-1}^{0}(2)\right)$ and $\lambda_{m}$ is the corresponding epimorphism for $m=n, n+1$. Then $\widetilde{s}$ induces the homomorphism

$$
\tilde{s}_{\kappa}^{*}: \mathbb{T}^{\kappa}(\mathcal{V}(n+1, n) ; G) \rightarrow \mathbb{T}^{\kappa}(\mathcal{V}(n, n-1) ; G)
$$

for any coefficient abelian group $G$.

Then we have the following. 
Proposition 5.5 Let $f_{i}: M_{i} \rightarrow N, i=0,1$, be $C^{0}$ stable $\tau$-maps of $n$-dimensional manifolds into an $(n-1)$-dimensional manifold $N$ which is a Thom map, where we assume that $M_{i}$ are closed. If they are $\tau$-cobordant, then for every $\kappa$, we have

$$
\tilde{\varphi}_{f_{0}}^{*} \circ \tilde{s}_{\kappa}^{*}=\tilde{\varphi}_{f_{1}}^{*} \circ \tilde{s}_{\kappa}^{*}: \mathbb{R}^{\kappa}(\mathcal{V}(n+1, n) ; G) \rightarrow H^{\kappa}(N ; G)
$$

for any coefficient abelian group $G$. In other words, we have

$$
\left.\tilde{\varphi}_{f_{0}}^{*}\right|_{\operatorname{Im} \tilde{s}_{\kappa}^{*}}=\left.\tilde{\varphi}_{f_{1}}^{*}\right|_{\operatorname{Im} \tilde{s}_{\kappa}^{*}}: \operatorname{Im} \widetilde{s}_{\kappa}^{*} \rightarrow H^{\kappa}(N ; G) .
$$

Proof Let $F: W \rightarrow N \times[0,1]$ be a $\tau$-cobordism between $f_{0}$ and $f_{1}$. Let us fix cell complex structures on $N$ and $N \times[0,1]$ which are compatible with each other. We denote by $C(N)$ and $C(N \times[0,1])$ the chain complexes (over the integers) of $N$ and $N \times[0,1]$ respectively associated with their cell complex structures. Then as in [10] we can construct chain maps

$$
\begin{aligned}
& \varphi_{f_{j}}: C(N) \rightarrow \mathcal{V}(n, n-1), \quad j=0,1, \quad \text { and } \\
& \varphi_{F}: C(N \times[0,1]) \rightarrow \mathcal{V}(n+1, n) .
\end{aligned}
$$

(For this, we do not need any free approximation.) Note that then there exist chain maps

$$
\begin{aligned}
& \widetilde{\varphi}_{f_{j}}: C(N) \rightarrow \mathcal{F}(n, n-1), \quad j=0,1, \quad \text { and } \\
& \tilde{\varphi}_{F}: C(N \times[0,1]) \rightarrow \mathcal{F}(n+1, n),
\end{aligned}
$$

unique up to chain homotopy, such that $\varphi_{f_{j}}=\lambda_{n} \circ \widetilde{\varphi}_{f_{j}}, j=0,1$, and $\varphi_{F}=\lambda_{n+1} \circ \widetilde{\varphi}_{F}$.

Now, let us consider the diagram of chain complexes as in Figure 4, where $i_{j \#}$ is the chain map induced by $i_{j}: N \rightarrow N \times[0,1]$ defined by $i_{j}(x)=(x, j), j=0,1$.

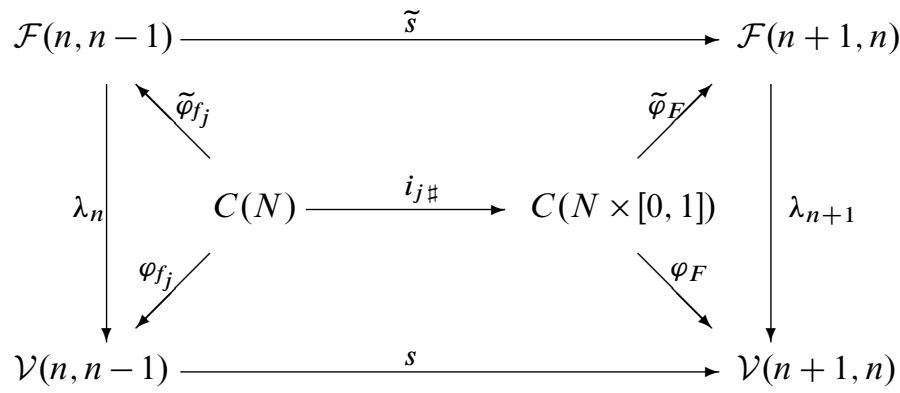

Figure 4: Diagram of chain complexes 
Note that we have

$$
\begin{aligned}
s \circ \lambda_{n} & =\lambda_{n+1} \circ \tilde{s} & \text { and } \\
s \circ \varphi_{f_{j}} & =\varphi_{F} \circ i_{j \sharp}, & j=0,1,
\end{aligned}
$$

where the latter equality follows from the constructions of $\varphi_{f_{j}}, \varphi_{F}$ and $s$. Therefore, we see that

$$
\lambda_{n+1} \circ\left(\tilde{s} \circ \tilde{\varphi}_{f_{j}}\right)=\lambda_{n+1} \circ \tilde{\varphi}_{F} \circ i_{j \#}
$$

holds for $j=0,1$. Then by the uniqueness of lifts up to chain homotopy (see [26, Chapter 2, Section 2, Lemma 13] and [10, Proposition 2.2]), we see that the chain maps $\tilde{s} \circ \widetilde{\varphi}_{f_{j}}$ and $\widetilde{\varphi}_{F} \circ i_{j \sharp}$ are chain homotopic.

Hence, they induce the identical homomorphisms in homology and cohomology. In particular, we have the commutative diagram

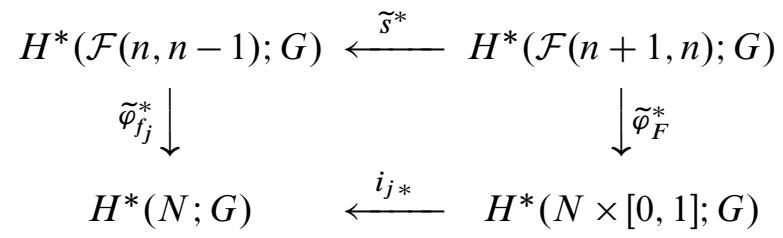

for any abelian group $G$. Since $i_{0}$ and $i_{1}$ are homotopic, we have $i_{0}^{*}=i_{1}^{*}$, and the result follows immediately.

Thus, it is expected that the hypercohomology of the universal homology complex of singular fibers gives more cobordism invariants than the usual cohomology of the universal cochain complex. However, in our situation, by virtue of Lemma 5.3, we get the same cobordism invariants.

It would be interesting to study the hypercohomologies of the higher dimensional analogues to see if there is an essential difference between the hypercohomologies and the usual cohomologies. If there is, then the corresponding cobordism invariant would lead to a "hidden singular fiber" in a sense similar to [10].

Remark 5.6 In this section, we have considered only $\tau$-maps (i.e. fold maps). However, this is not essential, and the same theory as in this section holds in the general framework as in [25].

\section{A topological invariant for map germs}

In this section, as an application of the theory of universal complexes of singular fibers as described in Sections 4 and 5, we give a new topological invariant for generic smooth map germs $\left(\mathbb{R}^{3}, 0\right) \rightarrow\left(\mathbb{R}^{2}, 0\right)$. 
In what follows, we will not distinguish a map germ from its representative when there is no confusion.

Definition 6.1 We say that a smooth map germ $g:\left(\mathbb{R}^{3}, 0\right) \rightarrow\left(\mathbb{R}^{2}, 0\right)$ is generic if for any sufficiently small positive real numbers $\varepsilon$ and $\delta$, the upper bound of $\delta$ depending on $g$ and the upper bound of $\varepsilon$ depending on $\delta$ and $g$, we have

(G1) $D_{\delta}^{3} \cap g^{-1}\left(S_{\varepsilon}^{1}\right)$ is a smooth manifold possibly with boundary,

(G2) $g_{\partial}=\left.g\right|_{D_{\delta}^{3} \cap g^{-1}\left(S_{\varepsilon}^{1}\right)}: D_{\delta}^{3} \cap g^{-1}\left(S_{\varepsilon}^{1}\right) \rightarrow S_{\varepsilon}^{1}$ is $C^{\infty}$ stable,

(G3) $\left.g\right|_{\partial D_{\delta}^{3} \cap g^{-1}\left(D_{\varepsilon}^{2}\right)}: \partial D_{\delta}^{3} \cap g^{-1}\left(D_{\varepsilon}^{2}\right) \rightarrow D_{\varepsilon}^{2}$ is a submersion, and

(G4) the restriction

$$
\left.g\right|_{D_{\delta}^{3} \cap g^{-1}\left(D_{\varepsilon}^{2} \backslash\{0\}\right)}: D_{\delta}^{3} \cap g^{-1}\left(D_{\varepsilon}^{2} \backslash\{0\}\right) \rightarrow D_{\varepsilon}^{2} \backslash\{0\}
$$

is proper, $C^{\infty}$ stable and $C^{\infty}$ equivalent to the product map

$$
g_{\partial} \times \operatorname{id}_{(0, \varepsilon)}:\left(D_{\delta}^{3} \cap g^{-1}\left(S_{\varepsilon}^{1}\right)\right) \times(0, \varepsilon) \rightarrow S_{\varepsilon}^{1} \times(0, \varepsilon)
$$

defined by $(x, t) \mapsto(g(x), t)$,

where $D_{\delta}^{3}$ (or $D_{\varepsilon}^{2}$ ) denotes the 3-dimensional ball in $\mathbb{R}^{3}$ (resp. 2-dimensional disk in $\mathbb{R}^{2}$ ) with radius $\delta$ (resp. $\varepsilon$ ) centered at the origin.

Note that the set of non-generic map germs has infinite codimension in an appropriate sense. For details, see the results of Fukuda [3] or Nishimura [15].

For a generic smooth map germ $g:\left(\mathbb{R}^{3}, 0\right) \rightarrow\left(\mathbb{R}^{2}, 0\right)$, set $U=D_{\delta}^{3} \cap g^{-1}\left(\operatorname{Int} D_{\varepsilon}^{2}\right)$ for $\delta$ and $\varepsilon$ as above. Note that $\left.g\right|_{U}: U \rightarrow \operatorname{Int} D^{2}$ is a proper smooth map. Let $\tilde{g}: U \rightarrow$ Int $D^{2} \subset \mathbb{R}^{2}$ be a proper $C^{\infty}$ stable perturbation of $\left.g\right|_{U}$ in a sense similar to that in $[4 ; 5 ; 16 ; 18]$.

For a cusp point $x \in U$ of $\tilde{g}$, we define its $\operatorname{sign} \operatorname{sign}(x) \in\{+1,-1\}$ as follows. Let $J_{0}(x)$ (resp. $\left.J_{1}(x)\right)$ be a short arc consisting of the definite fold points (resp. indefinite fold points) of $\tilde{g}$ near $x$. Then the arcs $\tilde{g}\left(J_{0}(x)\right)$ and $\tilde{g}\left(J_{1}(x)\right)$ are situated in $\mathbb{R}^{2}$ near $\tilde{g}(x)$ as depicted in Figure 5, and we define the sign as in the figure.

Note that by virtue of condition (G4) above, $\widetilde{g}$ has finitely many cusp points. The total number of cusp points of $\tilde{g}$, counted with signs, is called the algebraic number of cusps of $\tilde{g}$. 


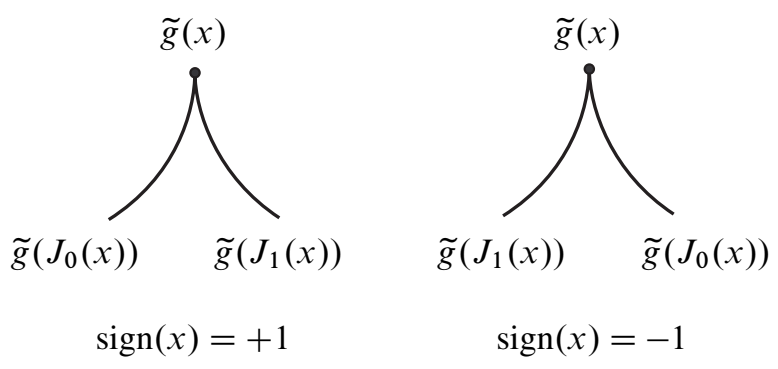

Figure 5: Sign for a cusp point $x$

Definition 6.2 Let $g$ and $g^{\prime}:\left(\mathbb{R}^{3}, 0\right) \rightarrow\left(\mathbb{R}^{2}, 0\right)$ be smooth map germs. We say that they are topologically $\mathcal{A}$-equivalent if there exist homeomorphism germs $\Phi:\left(\mathbb{R}^{3}, 0\right) \rightarrow$ $\left(\mathbb{R}^{3}, 0\right)$ and $\varphi:\left(\mathbb{R}^{2}, 0\right) \rightarrow\left(\mathbb{R}^{2}, 0\right)$ such that $g^{\prime}=\varphi^{-1} \circ g \circ \Phi$. Furthermore, if the homeomorphism germ $\varphi$ can be chosen so that it preserves the orientation of $\mathbb{R}^{2}$, then we say that $g$ and $g^{\prime}$ are topologically $\mathcal{A}_{+}$-equivalent.

The main result of this section is the following.

Theorem 6.3 Let $g:\left(\mathbb{R}^{3}, 0\right) \rightarrow\left(\mathbb{R}^{2}, 0\right)$ be a generic smooth map germ. Then the algebraic number of cusps of a $C^{\infty}$ stable perturbation $\tilde{g}$ of a representative of $g$ is an invariant of the topological $\mathcal{A}_{+}$-equivalence class of $g$. In particular, the absolute value of the algebraic number of cusps of $\tilde{g}$ is an invariant of the topological $\mathcal{A}$-equivalence class of $g$.

In order to prove the above theorem, let us first consider the following situation. Let $F: W \rightarrow D^{2}$ be a smooth map of a compact 3-dimensional manifold $W$ with nonempty boundary $\partial W=M$ with the following properties:

(1) $F^{-1}\left(\partial D^{2}\right)=M$,

(2) $f=\left.F\right|_{M}: M \rightarrow \partial D^{2}=S^{1}$ is $C^{\infty}$ stable,

(3) $\left.F\right|_{M \times[0,1)}=f \times \operatorname{id}_{[0,1)}$, where we identify the small open collar neighborhood of $M\left(\right.$ or $\left.\partial D^{2}\right)$ in $W\left(\right.$ resp. in $\left.D^{2}\right)$ with $M \times[0,1)\left(\right.$ resp. $\left.\partial D^{2} \times[0,1)\right)$, and

(4) $\left.F\right|_{\text {Int } W}:$ Int $W \rightarrow \operatorname{Int} D^{2}$ is a proper $C^{\infty}$ stable map.

Note that $F$ may have cusp singular points. Thus, in general, $F$ has singular fibers as depicted in Figure 3.

Algebraic ${ }^{6} \mathcal{G}$ Geometric Topology, Volume 6 (2006) 

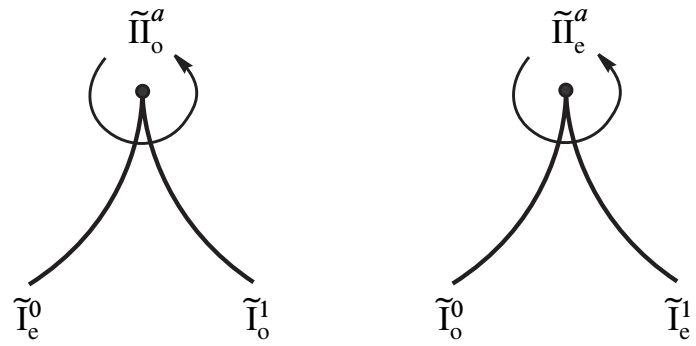

Figure 6: Co-orientations for $\widetilde{\mathrm{II}}_{\mathrm{o}}^{a}$ and $\widetilde{\mathrm{I}}_{\mathrm{e}}^{a}$

In Section 4 , we have seen that the fibers $\widetilde{\mathbf{0}}_{*}, \widetilde{\mathrm{I}}_{*}^{0}, \widetilde{\mathrm{I}}_{*}^{1}$ and $\widetilde{\mathrm{II}}_{*}^{01}$ are co-orientable. If cusp singular points are allowed, then we see easily that $\widetilde{\mathrm{I}}_{*}^{a}$ is also co-orientable. We give co-orientations to $\widetilde{\mathrm{II}}_{*}^{a}$ as depicted in Figure 6.

In the following, we orient $D^{2}$ and $S^{1}=\partial D^{2}$ consistently so that $S^{1}$ gets the counterclockwise orientation. Then we have the following.

Lemma 6.4 For the algebraic numbers of singular fibers of $F$ and $f$, we have the following:

$$
\begin{aligned}
& \left\|\widetilde{\mathrm{I}}_{\mathrm{o}}^{0}(f)\right\|=-\left\|\widetilde{\mathrm{I}}_{\mathrm{o}}^{01}(F)\right\|+\left\|\widetilde{\mathrm{I}}_{\mathrm{e}}^{01}(F)\right\|-\left\|\widetilde{\mathrm{I}}_{\mathrm{e}}^{a}(F)\right\|, \\
& \left\|\widetilde{\mathrm{I}}_{\mathrm{e}}^{0}(f)\right\|=-\left\|\widetilde{\mathrm{I}}_{\mathrm{o}}^{01}(F)\right\|+\left\|\widetilde{\mathrm{I}}_{\mathrm{e}}^{01}(F)\right\|+\left\|\widetilde{\mathrm{I}}_{\mathrm{o}}^{a}(F)\right\| \text {, } \\
& \left\|\widetilde{\mathrm{I}}_{\mathrm{o}}^{1}(f)\right\|=\left\|\widetilde{\mathrm{II}}_{\mathrm{o}}^{01}(F)\right\|-\left\|\widetilde{\mathrm{I}}_{\mathrm{e}}^{01}(F)\right\|-\left\|\widetilde{\mathrm{I}}_{\mathrm{o}}^{a}(F)\right\|, \\
& \left\|\widetilde{\mathrm{I}}_{\mathrm{e}}^{1}(f)\right\|=\left\|\widetilde{\mathrm{I}}_{\mathrm{o}}^{01}(F)\right\|-\left\|\widetilde{\mathrm{I}}_{\mathrm{e}}^{01}(F)\right\|+\left\|\widetilde{\mathrm{I}}_{\mathrm{e}}^{a}(F)\right\| \text {. }
\end{aligned}
$$

Proof Let us consider the closures of $\widetilde{\mathrm{I}}_{\mathrm{o}}^{0}(F), \widetilde{\mathrm{I}}_{\mathrm{e}}^{0}(F), \widetilde{\mathrm{I}}_{\mathrm{o}}^{1}(F)$ and $\widetilde{\mathrm{I}}_{\mathrm{e}}^{1}(F)$ as 1-dimensional chains in $D^{2}$ with coefficients in $\mathbb{Z}$. Then by observing the adjacencies for the singular fibers as we did to obtain the formulae for the coboundary homomorphism in (4-2), we get the following equalities as 0 -dimensional chains:

$$
\begin{aligned}
& \partial \overline{\widetilde{\mathrm{I}}_{\mathrm{o}}^{0}(F)}=\widetilde{\mathrm{II}}_{\mathrm{o}}^{01}(F)-\widetilde{\mathrm{II}}_{\mathrm{e}}^{01}(F)+\widetilde{\mathrm{I}}_{\mathrm{e}}^{a}(F)+\widetilde{\mathrm{I}}_{\mathrm{o}}^{0}(f), \\
& \partial \overline{\widetilde{\mathrm{I}}_{\mathrm{e}}^{0}(F)}=\widetilde{\mathrm{I}}_{\mathrm{o}}^{01}(F)-\widetilde{\mathrm{II}}_{\mathrm{e}}^{01}(F)-\widetilde{\mathrm{II}}_{\mathrm{o}}^{a}(F)+\widetilde{\mathrm{I}}_{\mathrm{e}}^{0}(f), \\
& \partial \widetilde{\widetilde{\mathrm{I}}_{\mathrm{o}}^{1}(F)}=-\widetilde{\mathrm{I}}_{\mathrm{o}}^{01}(F)+\widetilde{\mathrm{I}}_{\mathrm{e}}^{01}(F)+\widetilde{\mathrm{I}}_{\mathrm{o}}^{a}(F)+\widetilde{\mathrm{I}}_{\mathrm{o}}^{1}(f), \\
& \partial \overline{\widetilde{\mathrm{I}}_{\mathrm{e}}^{1}(F)}=-\widetilde{\mathrm{I}}_{\mathrm{o}}^{01}(F)+\widetilde{\mathrm{I}}_{\mathrm{e}}^{01}(F)-\widetilde{\mathrm{I}}_{\mathrm{e}}^{a}(F)+\widetilde{\mathrm{I}}_{\mathrm{e}}^{1}(f) .
\end{aligned}
$$

Since the algebraic number of points in the boundary of a 1-dimensional chain is always equal to zero, we get the desired equalities. 
Remark 6.5 By the above lemma, we see easily that

$$
\left\|\widetilde{\mathrm{I}}_{\mathrm{o}}^{0}(f)\right\|+\left\|\widetilde{\mathrm{I}}_{\mathrm{e}}^{1}(f)\right\|=0 \quad \text { and } \quad\left\|\widetilde{\mathrm{I}}_{\mathrm{e}}^{0}(f)\right\|+\left\|\widetilde{\mathrm{I}}_{\mathrm{o}}^{1}(f)\right\|=0 .
$$

This gives an alternative proof of the fact that $\beta_{1}(f)=0$ for a $C^{\infty}$ stable map $f$ of a closed surface into $S^{1}$ or into $\mathbb{R}\left(\subset S^{1}\right)$, where $\beta_{1}$ is the cohomology class described in Lemma 4.3 (see also [25, Lemma 14.1]).

For $\widetilde{\mathrm{II}}^{a}$, we consider the co-orientation as depicted in Figure 7. Since we have

$$
\left\|\widetilde{\mathrm{II}}^{a}(F)\right\|=\left\|\widetilde{\mathrm{II}}_{\mathrm{o}}^{a}(F)\right\|+\left\|\widetilde{\mathrm{I}}_{\mathrm{e}}^{a}(F)\right\|,
$$

we immediately get the following.

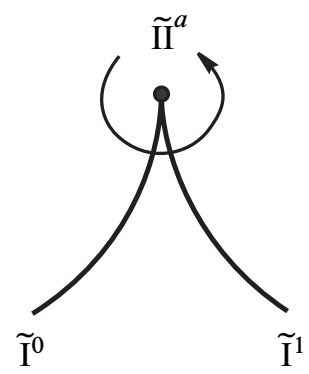

Figure 7: Co-orientation for $\widetilde{\mathrm{II}}^{a}$

Proposition 6.6 The algebraic number of singular fibers of $F$ containing cusps is equal to

$$
-\left\|\widetilde{\mathrm{I}}_{\mathrm{o}}^{0}(f)\right\|+\left\|\widetilde{\mathrm{I}}_{\mathrm{e}}^{0}(f)\right\|=-\left\|\widetilde{\mathrm{I}}_{\mathrm{o}}^{1}(f)\right\|+\left\|\widetilde{\mathrm{I}}_{\mathrm{e}}^{1}(f)\right\| .
$$

Note that the integer given by (6-1) is a fold cobordism invariant as shown in Section 4.

Now, let $g:\left(\mathbb{R}^{3}, 0\right) \rightarrow\left(\mathbb{R}^{2}, 0\right)$ be a generic smooth map germ. Suppose that the origin is isolated in $g^{-1}(0)$, i.e. $0 \notin \overline{g^{-1}(0) \backslash\{0\}}$. Then for $\varepsilon>0$ sufficiently small, $\widetilde{S}_{\varepsilon}^{2}=g^{-1}\left(S_{\varepsilon}^{1}\right)$ is diffeomorphic to $S^{2}$, and $\left.g\right|_{g^{-1}\left(D_{\varepsilon}^{2} \backslash\{0\}\right)}$ is $C^{\infty}$ equivalent to $g_{\partial} \times \operatorname{id}_{(0, \varepsilon)}$, where

$$
g_{\partial}=\left.g\right|_{g^{-1}\left(S_{\varepsilon}^{1}\right)}: \widetilde{S}_{\varepsilon}^{2} \rightarrow S_{\varepsilon}^{1}
$$

is a $C^{\infty}$ stable map.

Then, we have the following. 
Proposition 6.7 Let $g:\left(\mathbb{R}^{3}, 0\right) \rightarrow\left(\mathbb{R}^{2}, 0\right)$ be a generic smooth map germ such that 0 is isolated in $g^{-1}(0)$. Then the algebraic number of cusps of a $C^{\infty}$ stable perturbation $\tilde{g}$ of a representative of $g$ is an invariant of the topological $\mathcal{A}_{+}$-equivalence class of $g$, and is equal to

$$
-\left\|\widetilde{\mathrm{I}}_{\mathrm{o}}^{0}\left(g_{\partial}\right)\right\|+\left\|\widetilde{\mathrm{I}}_{\mathrm{e}}^{0}\left(g_{\partial}\right)\right\|=-\left\|\widetilde{\mathrm{I}}_{\mathrm{o}}^{1}\left(g_{\partial}\right)\right\|+\left\|\widetilde{\mathrm{I}}_{\mathrm{e}}^{1}\left(g_{\partial}\right)\right\|,
$$

where

$$
g_{\partial}=\left.g\right|_{g^{-1}\left(S_{\varepsilon}^{1}\right)}: \widetilde{S}_{\varepsilon}^{2} \rightarrow S_{\varepsilon}^{1}
$$

and $\varepsilon>0$ is sufficiently small. In particular, the absolute value of the algebraic number of cusps of $\tilde{g}$ is an invariant of the topological $\mathcal{A}$-equivalence class of $g$.

For the proof, we need the following observation.

Remark 6.8 The notion of a singular fiber and the corresponding $C^{0}$ equivalence relation can be generalized to continuous maps between topological spaces. In particular, the theory as developed in [25] can be generalized to proper continuous maps between topological manifolds which are topologically equivalent to smooth Thom maps between smooth manifolds, and their topological cobordisms. This is because the classification of singular fibers is based on the " $C^{0}$ equivalence" and not on the " $C^{\infty}$ equivalence" in the theory developed in [25].

Proof of Proposition 6.7 Let $g^{\prime}:\left(\mathbb{R}^{3}, 0\right) \rightarrow\left(\mathbb{R}^{2}, 0\right)$ be a smooth map germ which is generic and is topologically $\mathcal{A}_{+}$-equivalent to $g$. Thus there exist homeomorphism germs $\Phi:\left(\mathbb{R}^{3}, 0\right) \rightarrow\left(\mathbb{R}^{3}, 0\right)$ and $\varphi:\left(\mathbb{R}^{2}, 0\right) \rightarrow\left(\mathbb{R}^{2}, 0\right)$ such that $g^{\prime}=\varphi^{-1} \circ g \circ \Phi$ and $\varphi$ is orientation preserving.

Let $\varepsilon$ (resp. $\varepsilon^{\prime}$ ) be a small positive real number as above for $g$ (resp. for $g^{\prime}$ ). We can take $\varepsilon^{\prime}$ sufficiently small so that $\varphi\left(D_{\varepsilon^{\prime}}^{2}\right) \subset \operatorname{Int} D_{\varepsilon}^{2}$. Set

$$
Y=D_{\varepsilon}^{2} \backslash \varphi\left(\operatorname{Int} D_{\varepsilon^{\prime}}^{2}\right) \quad \text { and } \quad W=g^{-1}(Y) \text {. }
$$

We can show that $Y$ is homeomorphic to $S^{1} \times[0,1]$ and $W$ is homeomorphic to $S^{2} \times[0,1]$. Note that $g^{-1}\left(\varphi\left(S_{\mathcal{\varepsilon}^{\prime}}^{1}\right)\right)=\Phi\left(g^{-1}\left(S_{\mathcal{\varepsilon}^{\prime}}^{1}\right)\right)$.

Then the map $\left.g\right|_{W}: W \rightarrow Y$ gives a (fold) cobordism between

$$
g_{\partial}=\left.g\right|_{g^{-1}\left(S_{\varepsilon}^{1}\right)}: g^{-1}\left(S_{\varepsilon}^{1}\right) \rightarrow S_{\varepsilon}^{1}
$$

and

$$
\varphi \circ g_{\partial}^{\prime} \circ \Phi^{-1}: \Phi\left(g^{\prime-1}\left(S_{\varepsilon^{\prime}}^{1}\right)\right) \rightarrow \varphi\left(S_{\mathcal{\varepsilon}^{\prime}}^{1}\right) .
$$


(Precisely speaking, $W$ and $Y$ are only topological manifolds with boundary and $\left.g\right|_{W}$ is merely a continuous map. Nevertheless, we can regard $\left.g\right|_{W}$ as a topological fold cobordism in an appropriate sense. See Remark 6.8.)

Then by the results obtained in Section 4 , we have ${ }^{5}$

$$
-\left\|\widetilde{\mathrm{I}}_{\mathrm{o}}^{0}\left(g_{\partial}\right)\right\|+\left\|\widetilde{\mathrm{I}}_{\mathrm{e}}^{0}\left(g_{\partial}\right)\right\|=-\left\|\widetilde{\mathrm{I}}_{\mathrm{o}}^{0}\left(g_{\partial}^{\prime}\right)\right\|+\left\|\widetilde{\mathrm{I}}_{\mathrm{e}}^{0}\left(g_{\partial}^{\prime}\right)\right\| .
$$

This is because $\varphi$ preserves the orientation of $\mathbb{R}^{2}$ and hence the algebraic number of singular fibers of a given type for the map (6-3) is equal to that for $g_{\partial}^{\prime}$. Therefore, the integer (6-2) is an invariant of the topological $\mathcal{A}_{+}$-equivalence class. (Note that the equality in (6-2) follows from Proposition 6.6.)

On the other hand, let $\tilde{g}: U \rightarrow \operatorname{Int} D_{\varepsilon}^{2}$ be a proper $C^{\infty}$ stable perturbation of $\left.g\right|_{U}$, where $U=g^{-1}\left(\operatorname{Int} D_{\varepsilon}^{2}\right)$. Set

$$
U^{\prime}=g^{-1}\left(\operatorname{Int} D_{\varepsilon}^{2} \backslash D_{\varepsilon / 2}^{2}\right) \quad \text { and } \quad U^{\prime \prime}=\tilde{g}^{-1}\left(\operatorname{Int} D_{\varepsilon}^{2} \backslash D_{\varepsilon / 2}^{2}\right) .
$$

Then, by virtue of conditions (G2) and (G4) of Definition 6.1, we see that $\left.g\right|_{U^{\prime}}$ and $\left.\tilde{g}\right|_{U^{\prime \prime}}$ are topologically $\mathcal{A}_{+}$-equivalent in a sense similar to Definition 6.2. Then we see that the integer (6-2) is equal to the algebraic number of cusps of $\widetilde{g}$ by Proposition 6.6 .

It is easy to observe that if we reverse the orientation of $\mathbb{R}^{2}$, then the algebraic number of cusps changes the sign. Thus the last assertion of Proposition 6.7 follows immediately. This completes the proof.

In order to generalize the above result to the case where the origin may not necessarily be isolated in $g^{-1}(0)$, let us consider the following situation. Let $F: W \rightarrow D^{2}$ be a smooth map of a compact 3-dimensional manifold $W$ with nonempty boundary $\partial W$ with the following properties:

(1) $\partial W=M \cup P$, where $M$ is a compact surface with boundary, $P$ is a finite disjoint union of 2-dimensional disks, and $M \cap P=\partial M=\partial P$,

(2) $F^{-1}\left(\partial D^{2}\right)=M$,

(3) $\left.F\right|_{P}: P \rightarrow D^{2}$ is a submersion so that, in particular, it is a diffeomorphism on each component of $P$,

(4) $f=\left.F\right|_{M}: M \rightarrow \partial D^{2}=S^{1}$ is $C^{\infty}$ stable,

(5) $\left.F\right|_{M \times[0,1)}=f \times \mathrm{id}_{[0,1)}$, where we identify the small open "collar neighborhood" of $M\left(\right.$ or $\left.\partial D^{2}\right)$ in $W\left(\right.$ resp. in $\left.D^{2}\right)$ with $M \times[0,1)\left(\right.$ resp. $\left.\partial D^{2} \times[0,1)\right)$, and

\footnotetext{
${ }^{5}$ In Section 4 we have considered Morse functions on surfaces: however, almost all the arguments work also for $C^{\infty}$ stable maps into $S^{1}$.
} 
(6) $\left.F\right|_{W \backslash M}: W \backslash M \rightarrow \operatorname{Int} D^{2}$ is a proper $C^{\infty}$ stable map.

In what follows, for simplicity we assume that $W$ and $M$ are orientable, which is enough for our purpose.

Then we can get a list of the $C^{0}$ equivalence classes of singular fibers that appear for $F$ as above, which is similar to Figure 3. For these fibers, let us consider the following equivalence relation: two fibers are equivalent if one is $C^{0}$ equivalent to the other one after adding even numbers of regular components to both of the fibers. Note that in contrast to the case where $\partial M=\varnothing$, regular fibers consist of circles and intervals. However, when we count the number of regular components, we do not distinguish them.

Then we easily get the following.

Lemma 6.9 Those equivalence classes of singular fibers which are co-orientable are $\widetilde{\mathfrak{F}}_{*}$, where $*=\mathrm{o}$ or e, and $\widetilde{\mathfrak{F}}$ are as depicted in Figure 8.

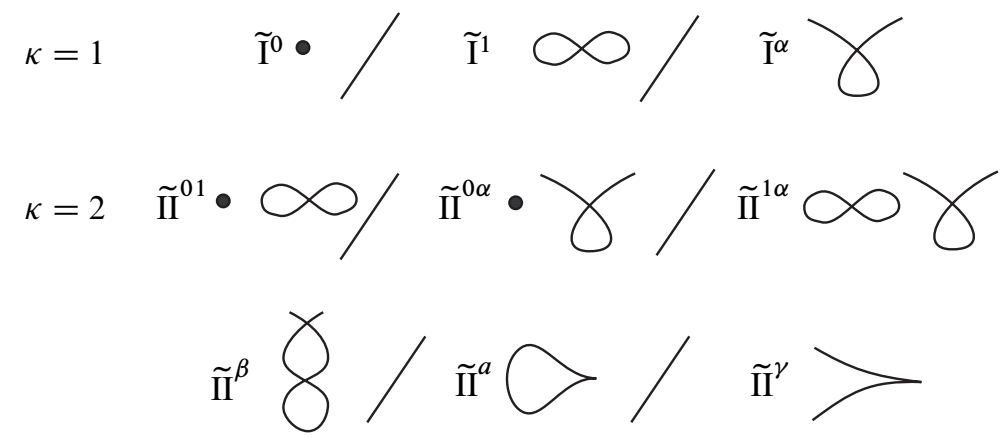

Figure 8: List of co-orientable singular fibers for $F$

We denote by $\tilde{\mathbf{0}}_{*}$ the equivalence classes corresponding to regular fibers. Note that they are also co-orientable. We fix a co-orientation for each co-orientable equivalence class of singular fibers as above. Then for the coboundary homomorphism, we get the following:

$$
\begin{aligned}
& \delta_{1}\left(\widetilde{\mathrm{I}}_{\mathrm{o}}^{0}\right)=\widetilde{\mathrm{II}}_{\mathrm{o}}^{01}-\widetilde{\mathrm{II}}_{\mathrm{e}}^{01}-\widetilde{\mathrm{II}}_{\mathrm{e}}^{a}-\widetilde{\mathrm{II}}_{\mathrm{o}}^{0 \alpha}+\widetilde{\mathrm{II}}_{\mathrm{e}}^{0 \alpha}-\widetilde{\mathrm{II}}_{\mathrm{e}}^{\gamma}, \\
& \delta_{1}\left(\widetilde{\mathrm{I}}_{\mathrm{e}}^{0}\right)=\widetilde{\mathrm{II}}_{\mathrm{o}}^{01}-\widetilde{\mathrm{I}}_{\mathrm{e}}^{01}+\widetilde{\mathrm{II}}_{\mathrm{o}}^{a}-\widetilde{\mathrm{II}}_{\mathrm{o}}^{0 \alpha}+\widetilde{\mathrm{II}}_{\mathrm{e}}^{0 \alpha}+\widetilde{\mathrm{I}}_{\mathrm{o}}^{\gamma}, \\
& \delta_{1}\left(\widetilde{\mathrm{I}}_{\mathrm{o}}^{1}\right)=-\widetilde{\mathrm{I}}_{\mathrm{o}}^{01}+\widetilde{\mathrm{II}}_{\mathrm{e}}^{01}-\widetilde{\mathrm{II}}_{\mathrm{o}}^{a}-\widetilde{\mathrm{II}}_{\mathrm{o}}^{1 \alpha}+\widetilde{\mathrm{II}}_{\mathrm{e}}^{1 \alpha}-\widetilde{\mathrm{II}}_{\mathrm{e}}^{\beta},
\end{aligned}
$$




$$
\begin{aligned}
& \delta_{1}\left(\widetilde{\mathrm{I}}_{\mathrm{e}}^{1}\right)=-\widetilde{\mathrm{I}}_{\mathrm{o}}^{01}+\widetilde{\mathrm{I}}_{\mathrm{e}}^{01}+\widetilde{\mathrm{II}}_{\mathrm{e}}^{\alpha}-\widetilde{\mathrm{II}}_{\mathrm{o}}^{1 \alpha}+\widetilde{\mathrm{II}}_{\mathrm{e}}^{1 \alpha}+\widetilde{\mathrm{II}}_{\mathrm{o}}^{\beta}, \\
& \left.\delta_{1} \widetilde{\mathrm{I}}_{\mathrm{o}}^{\alpha}\right)=\widetilde{\mathrm{II}}_{\mathrm{o}}^{0 \alpha}-\widetilde{\mathrm{I}}_{\mathrm{e}}^{0 \alpha}+\widetilde{\mathrm{II}}_{\mathrm{o}}^{1 \alpha}-\widetilde{\mathrm{I}}_{\mathrm{e}}^{1 \alpha}+\widetilde{\mathrm{I}}_{\mathrm{e}}^{\beta}-\widetilde{\mathrm{I}}_{\mathrm{o}}^{\gamma}, \\
& \left.\delta_{1} \widetilde{\mathrm{I}}_{\mathrm{e}}^{\alpha}\right)=\widetilde{\mathrm{II}}_{\mathrm{o}}^{0 \alpha}-\widetilde{\mathrm{I}}_{\mathrm{e}}^{0 \alpha}+\widetilde{\mathrm{II}}_{\mathrm{o}}^{1 \alpha}-\widetilde{\mathrm{II}}_{\mathrm{e}}^{1 \alpha}-\widetilde{\mathrm{II}}_{\mathrm{o}}^{\beta}+\widetilde{\mathrm{I}}_{\mathrm{e}}^{\gamma} .
\end{aligned}
$$

Then by the same argument as before, we see that

$(6-4) \quad\left\|\widetilde{\mathrm{I}}_{\mathrm{o}}^{\alpha}(f)\right\|-\left\|\widetilde{\mathrm{I}}_{\mathrm{e}}^{\alpha}(f)\right\|+\left\|\widetilde{\mathrm{I}}_{\mathrm{o}}^{1}(f)\right\|-\left\|\widetilde{\mathrm{I}}_{\mathrm{e}}^{1}(f)\right\| \quad$ and $\quad-\left\|\widetilde{\mathrm{I}}_{\mathrm{o}}^{0}(f)\right\|+\left\|\widetilde{\mathrm{I}}_{\mathrm{e}}^{0}(f)\right\|$

are fold cobordism invariants of $f$ in the following sense.

Definition 6.10 Let $f_{i}: M_{i} \rightarrow S^{1}, i=0,1$, be proper $C^{\infty}$ stable maps of compact surfaces with boundary such that $\left.f_{i}\right|_{\partial M_{i}}$ are submersions. We say that $f_{0}$ and $f_{1}$ are (fold) cobordant if there exist a compact 3-dimensional manifold $X$ with corners and a fold map $F: X \rightarrow S^{1} \times[0,1]$ with the following properties:

(1) $\partial X=M_{0} \cup Q \cup M_{1}$, where $M_{0} \cap M_{1}=\varnothing$ and $Q$ is a compact surface with boundary $\partial Q=\left(Q \cap M_{0}\right) \cup\left(Q \cap M_{1}\right)$,

(2) $X$ has corners along $\partial Q$,

(3) $\left.F\right|_{Q}: Q \rightarrow S^{1} \times[0,1]$ is a submersion, and

(4) we have

$$
\begin{aligned}
\left.F\right|_{M_{0} \times[0, \varepsilon)} & =f_{0} \times \operatorname{id}_{[0, \varepsilon)}: M_{0} \times[0, \varepsilon) \rightarrow S^{1} \times[0, \varepsilon), \quad \text { and } \\
\left.F\right|_{M_{1} \times(1-\varepsilon, 1]} & =f_{1} \times \operatorname{id}_{(1-\varepsilon, 1]}: M_{1} \times(1-\varepsilon, 1] \rightarrow S^{1} \times(1-\varepsilon, 1]
\end{aligned}
$$

for some sufficiently small $\varepsilon>0$, where we identify the open "collar neighborhoods" of $M_{0}$ and $M_{1}$ in $X$ with $M_{0} \times[0, \varepsilon)$ and $M_{1} \times(1-\varepsilon, 1]$ respectively.

Furthermore, we see that the algebraic number of cusps of $F$ given by

$$
\left\|\widetilde{\mathrm{II}}_{\mathrm{o}}^{a}(F)\right\|+\left\|\widetilde{\mathrm{II}}_{\mathrm{e}}^{a}(F)\right\|+\left\|\tilde{\mathrm{II}}_{\mathrm{o}}^{\gamma}(F)\right\|+\left\|\tilde{\mathrm{I}}_{\mathrm{e}}^{\gamma}(F)\right\|
$$

is equal to both of the integers (6-4).

Now, let $g:\left(\mathbb{R}^{3}, 0\right) \rightarrow\left(\mathbb{R}^{2}, 0\right)$ be a generic smooth map germ. Then by applying the above observations to the stable perturbation of the map

$$
\left.g\right|_{D_{\delta}^{3} \cap g^{-1}\left(\operatorname{Int} D_{\varepsilon}^{2}\right)}: D_{\delta}^{3} \cap g^{-1}\left(\operatorname{Int} D_{\varepsilon}^{2}\right) \rightarrow \operatorname{Int} D_{\varepsilon}^{2},
$$

we get the following, where $\delta$ and $\varepsilon$ are as in Definition 6.1. 
Proposition 6.11 Let $g:\left(\mathbb{R}^{3}, 0\right) \rightarrow\left(\mathbb{R}^{2}, 0\right)$ be a generic smooth map germ. Then the algebraic number of cusps of a $C^{\infty}$ stable perturbation $\tilde{g}$ of a representative of $g$ is an invariant of the topological $\mathcal{A}_{+}$-equivalence class of $g$, and is equal to

$$
-\left\|\widetilde{I}_{\mathrm{o}}^{0}\left(g_{\partial}\right)\right\|+\left\|\widetilde{I}_{\mathrm{e}}^{0}\left(g_{\partial}\right)\right\|=\left\|\widetilde{\mathrm{I}}_{\mathrm{o}}^{\alpha}\left(g_{\partial}\right)\right\|-\left\|\widetilde{\mathrm{I}}_{\mathrm{e}}^{\alpha}\left(g_{\partial}\right)\right\|+\left\|\widetilde{\mathrm{I}}_{\mathrm{o}}^{1}\left(g_{\partial}\right)\right\|-\left\|\widetilde{\mathrm{I}}_{\mathrm{e}}^{1}\left(g_{\partial}\right)\right\|,
$$

where

$$
g_{\partial}=\left.g\right|_{D_{\delta}^{3} \cap g^{-1}\left(S_{\varepsilon}^{1}\right)}: D_{\delta}^{3} \cap g^{-1}\left(S_{\varepsilon}^{1}\right) \rightarrow S_{\varepsilon}^{1}
$$

and $0<\varepsilon<<\delta$ are sufficiently small. In particular, the absolute value of the algebraic number of cusps of $\widetilde{g}$ is an invariant of the topological $\mathcal{A}$-equivalence class of $g$.

Proof Let $g^{\prime}:\left(\mathbb{R}^{3}, 0\right) \rightarrow\left(\mathbb{R}^{2}, 0\right)$ be a smooth map germ which is generic and is topologically $\mathcal{A}_{+}$-equivalent to $g$. Thus there exist homeomorphism germs $\Phi:\left(\mathbb{R}^{3}, 0\right) \rightarrow$ $\left(\mathbb{R}^{3}, 0\right)$ and $\varphi:\left(\mathbb{R}^{2}, 0\right) \rightarrow\left(\mathbb{R}^{2}, 0\right)$ such that $g^{\prime}=\varphi^{-1} \circ g \circ \Phi$ and $\varphi$ is orientation preserving.

Let $\delta$ and $\varepsilon$ (resp. $\delta^{\prime}$ and $\varepsilon^{\prime}$ ) be small positive real numbers as in Definition 6.1 for $g$ (resp. for $g^{\prime}$ ). We can take $\delta^{\prime}$ and $\varepsilon^{\prime}$ sufficiently small so that $\varphi\left(D_{\varepsilon^{\prime}}^{2}\right) \subset \operatorname{Int} D_{\varepsilon}^{2}$ and $\Phi\left(g^{\prime-1}\left(D_{\varepsilon^{\prime}}^{2}\right) \cap D_{\delta^{\prime}}^{3}\right) \subset g^{-1}\left(\operatorname{Int} D_{\varepsilon}^{2}\right) \cap \operatorname{Int} D_{\delta}^{3}$.

Set

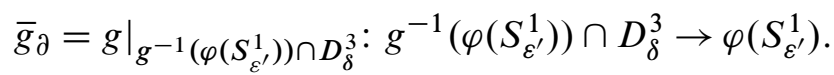

It is easy to observe that for each $y \in \varphi\left(S_{\varepsilon^{\prime}}^{1}\right)$, the fiber of $\bar{g}_{\partial}$ over $y$ and that of

$$
\varphi \circ g_{\partial}^{\prime} \circ \Phi^{-1}: \Phi\left(g^{\prime-1}\left(S_{\varepsilon^{\prime}}^{1}\right) \cap D_{\delta^{\prime}}^{3}\right) \rightarrow \varphi\left(S_{\varepsilon^{\prime}}^{1}\right)
$$

over $y$ is $C^{0}$ equivalent.

Set $Y=D_{\varepsilon}^{2} \backslash \varphi\left(\operatorname{Int} D_{\varepsilon^{\prime}}^{2}\right)$ and $W=g^{-1}(Y) \cap D_{\delta}^{3}$. We see that $Y$ is homeomorphic to $S^{1} \times[0,1]$ and $\left.g\right|_{W}: W \rightarrow Y$ gives a (fold) cobordism between $g_{\partial}$ and $\bar{g}_{\partial}$ in the sense of Definition 6.10 (see also Remark 6.8). (Note that the boundary causes no problem by virtue of condition (G3) of Definition 6.1.)

Then by the same argument as in the proof of Proposition 6.7 together with the above results, we get the desired result.

Now Theorem 6.3 follows from Propositions 6.7 and 6.11 .

Compare Theorem 6.3 with the results obtained in $[4 ; 5 ; 16 ; 18]$, etc. It would be an interesting problem to find a formula expressing the algebraic number of cusps of a $C^{\infty}$ stable perturbation in algebraic terms: e.g. as the signature of a certain quadratic form associated with a generic map germ. 
It would also be interesting to find topological invariants of generic smooth map germs $\left(\mathbb{R}^{n}, 0\right) \rightarrow\left(\mathbb{R}^{p}, 0\right)$ with $n>p$ arising from the number of certain singular fibers of a stable (or Thom-Boardman generic) perturbation.

\section{References}

[1] A du Plessis, T Wall, The geometry of topological stability, London Mathematical Society Monographs. New Series 9, The Clarendon Press, Oxford University Press, New York (1995) MR1408432

[2] A T Fomenko, Differential geometry and topology, Contemporary Soviet Mathematics, Consultants Bureau, New York (1987) MR893812

[3] T Fukuda, Local topological properties of differentiable mappings. II, Tokyo J. Math. 8 (1985) 501-520 MR827005

[4] T Fukuda, G Ishikawa, On the number of cusps of stable perturbations of a plane-toplane singularity, Tokyo J. Math. 10 (1987) 375-384 MR926249

[5] T Fukui, J J Nuño Ballesteros, M J Saia, On the number of singularities in generic deformations of map germs, J. London Math. Soc. (2) 58 (1998) 141-152 MR1666098

[6] M Golubitsky, V Guillemin, Stable mappings and their singularities, Springer-Verlag, New York (1973) MR0341518

[7] K Ikegami, Cobordism group of Morse functions on manifolds, Hiroshima Math. J. 34 (2004) 211-230 MR2086843

[8] K Ikegami, O Saeki, Cobordism group of Morse functions on surfaces, J. Math. Soc. Japan 55 (2003) 1081-1094 MR2003761

[9] B Kalmár, Cobordism group of Morse functions on unoriented surfaces, Kyushu J. Math. 59 (2005) 351-363 MR2188598

[10] M È Kazaryan, Hidden singularities and Vassiliev's homology complex of singularity classes, Mat. Sb. 186 (1995) 119-128 MR1376094 English translation in Sb. Math. 186 (1995) 1811-1820

[11] M A Kervaire, J W Milnor, Groups of homotopy spheres. I, Ann. of Math. (2) 77 (1963) 504-537 MR0148075

[12] L Kushner, H Levine, P Porto, Mapping three-manifolds into the plane. I, Bol. Soc. Mat. Mexicana (2) 29 (1984) 11-33 MR790729

[13] H Levine, Elimination of cusps, Topology 3 (1965) 263-296 MR0176484

[14] H Levine, Classifying immersions into $\mathbf{R}^{4}$ over stable maps of 3-manifolds into $\mathbf{R}^{2}$, Lecture Notes in Mathematics 1157, Springer-Verlag, Berlin (1985) MR814689

[15] T Nishimura, Topologically $\infty$-determined map germs are topologically cone-like, Compositio Math. 64 (1987) 117-129 MR911358 
[16] J J Nuño Ballesteros, M J Saia, Multiplicity of Boardman strata and deformations of map germs, Glasgow Math. J. 40 (1998) 21-32 MR1612145

[17] T Ohmoto, Vassiliev complex for contact classes of real smooth map-germs, Rep. Fac. Sci. Kagoshima Univ. Math. Phys. Chem. 27 (1994) 1-12 MR1341346

[18] M Ohsumi, Whitney's umbrellas in stable perturbations of a map germ from $\left(\mathbb{R}^{n}, 0\right) \rightarrow$ $\left(\mathbb{R}^{2 n-1}, 0\right)$, preprint (2004)

[19] R Rimányi, A Szücs, Pontrjagin-Thom-type construction for maps with singularities, Topology 37 (1998) 1177-1191 MR1632908

[20] R Sadykov, Bordism groups of special generic mappings, Proc. Amer. Math. Soc. 133 (2005) 931-936 MR2113946

[21] O Saeki, Simple stable maps of 3-manifolds into surfaces. II, J. Fac. Sci. Univ. Tokyo Sect. IA Math. 40 (1993) 73-124 MR1217661

[22] O Saeki, Topology of special generic maps into $\mathbf{R}^{3}$, Mat. Contemp. 5 (1993) 161-186 MR1305180

[23] O Saeki, Simple stable maps of 3-manifolds into surfaces, Topology 35 (1996) 671-698 MR1396772

[24] O Saeki, Cobordism groups of special generic functions and groups of homotopy spheres, Japan. J. Math. (N.S.) 28 (2002) 287-297 MR1947905

[25] O Saeki, Topology of singular fibers of differentiable maps, Lecture Notes in Mathematics 1854, Springer-Verlag, Berlin (2004) MR2106689

[26] EH Spanier, Algebraic topology, McGraw-Hill Book Co., New York (1966) MR0210112

[27] R Thom, Quelques propriétés globales des variétés différentiables, Comment. Math. Helv. 28 (1954) 17-86 MR0061823

[28] V A Vassilyev, Lagrange and Legendre characteristic classes, Advanced Studies in Contemporary Mathematics 3, Gordon and Breach Science Publishers, New York (1988) MR1065996

Faculty of Mathematics, Kyushu University

Hakozaki, Fukuoka 812-8581, Japan

saeki@math.kyushu-u.ac.jp

http://www.math.kyushu-u.ac.jp/ saeki/

Received: 22 September 2005 